\title{
Affine quotients of supergroups
}

\author{
A.N.Zubkov
}

\begin{abstract}
In this article we consider sheaf quotients of affine superschemes by affine supergroups that act on them freely. The necessary and sufficient conditions for such quotients to be affine are given. If $G$ is an affine supergroup and $H$ is its normal supersubgroup, then we prove that a dur $K$-sheaf $G \tilde{\tilde{}} H$ is again affine supergroup. Additionally, if $G$ is algebraic, then a $K$-sheaf $G \tilde{/} H$ is also algebraic supergroup and it coincides with $G \tilde{\tilde{}} H$. In particular, any normal supersubgroup of an affine supergroup is faithfully exact.
\end{abstract}

\section{Introduction}

Let $G$ be an algebraic group defined over an algebraically closed field $K$ of an arbitrary characteristic. A closed subgroup $H$ of $G$ is called exact iff the induced functor $i n d_{H}^{G}$ is exact. A remarkable theorem of Cline-Parshall-Scott says that $H$ is exact iff the quotient $G / H$ is affine iff $K[G]$ is an injective $H$-module [21. In the category of affine schemes, wellknown Takeuchi's theorem states that the dur $K$-sheaf (faisceau dur in the terminology from [6]) of right cosets $G \tilde{\tilde{/}} H$ is affine iff $K[G]$ is a faithfully coflat right (or/and left, respectively) $K[H]$-comodule iff $K[G]$ is an injective cogenerator in the category of right (or/and left, respectively) $K[H]$-comodules [12, 14]. In this case $H$ is called faithfully exact.

In the category of affine supergroups, only the second equivalence of the above ClineParshall-Scott theorem has been recently proved in [1. The definition of a dur $K$-sheaf (as well as the definition of a $K$-sheaf or faisceau, see [6]) can be easily adapted to the category of $K$-functors over commutative superalgebras. Therefore, it is natural to ask whether, for an affine supergroup $G$ and its supersubgroup $H$, the dur $K$-sheaf $G \tilde{\tilde{/}} H$ is affine. In the case when $G$ is algebraic, that is $K[G]$ is a finitely generated superalgebra, one can also ask whether the $K$-sheaf $G \tilde{/} H$ is affine. Notice that in general we only have inclusion $G \tilde{/} H \subseteq G \tilde{\tilde{F}} H$ but if $G \tilde{/} H$ is an affine superscheme, then $G \tilde{/} H=G \tilde{\tilde{/}} H$.

In the present article we consider more general case when $G$ acts on an affine superscheme $X$ freely and we give necessary and sufficient conditions for $X \tilde{\tilde{/}} G$ (and $X \tilde{X} G$, respectively) to be an affine superscheme. Following [10, 12, 14, we generalize some theorems about Hopf algebras and their coideal subalgebras to Hopf superalgebras and their coideal supersubalgebras. In particular, we obtain a superalgebra version of the above Takeuchi's theorem.

Equipped with these results, we proceed to prove the main theorem of our article. Namely, the fundamental theorem of the algebraic group theory states that if $G$ is an 
algebraic group and $H$ is its closed normal subgroup, then $G / H$ is again an algebraic group, see [6, 17, 27. For any affine supergroup $G$ and its closed normal supersubgroup $H$ we prove that $G \tilde{\tilde{}} H$ is again an affine supergroup. Moreover, if $G$ is algebraic, then $G \tilde{\tilde{/}} H=G \tilde{/} H$ is also algebraic. It follows that any closed normal supersubgroup of an affine supergroup is faithfully exact.

The article is organized as follows. In the first section necessary definitions and notations concerning super(co)algebras and super(co)modules over them are collected. On top of that, (co)flat and faithfully (co)flat super(co)modules are defined and some well-known results about flat and faithfully flat modules over commutative algebras are generalized to (super)modules over commutative superalgebras. In the second section we introduce $K$-functors as functors from the category of commutative superalgebras to the category of sets. More attention is paid to the particular case of affine superschemes. We also define (dur) $K$-sheafs and completions of certain $K$-functors in the Grothendieck topology of faithfully flat (faithfully flat and finitely presented) coverings. In the third section we define the superspace of distributions of an affine superscheme. This superspace has a natural structure of (cocommutative) Hopf superalgebra provided the original superscheme is a supergroup. Moreover, if char $K=0$ and this supergroup is algebraic, then we prove that the corresponding distribution superalgebra is isomorphic to the universal enveloping superalgebra of its Lie superalgebra. In addition, we introduce the notion of Lie superalgebra functor by means of superalgebra of dual numbers (see [29]).

In the fourth section we consider an affine superscheme $X$ and a supergroup $G$ that acts on $X$ (on the right) freely. We give necessary and sufficient conditions for a quotient $X \tilde{\tilde{/}} G(X \tilde{/} G)$ to be affine. The main results of the fifth section are Theorem 5.1 and 5.2. Theorem 5.1 says surprisingly more about Hopf supersubalgebras than one would expect. Keeping in mind Takeuchi's theorem it would be quite natural to expect that a Hopf superalgebra is (left and right) faithfully flat supermodule over its Hopf supersubalgebra but it is actually faithfully flat as a module!

In sixth and ninth sections we prove the main result of this article. The principal idea is different from [11, 17] for the following reason. In the category of vector superspaces, an exterior power does not have the same nice properties as in the category of vector spaces. More precisely, if $V$ is a superspace of finite (super)dimension $(m, n)$ and $W$ is its supersubspace of (super)dimension $(s, t)$, where $t>0$, then no exterior power $\Lambda^{d}(W)$ is one-dimensional. In particular, the "naive" or "direct" generalization of Chevalley's theorem is not possible. Instead of exterior powers one can use a berezinian $\operatorname{Ber}_{s \mid t}(W)$ of $W$ but it does not solve our problem. In fact, we would have to embed $\operatorname{Ber}_{s, t}(W)$ to something like $\operatorname{Ber}_{s, t}(V)$ (analogously as $\Lambda^{k}(U)$ is embedded into $\Lambda^{k}(L)$, where $U$ is a subspace of a space $L$ and $k=\operatorname{dim} U$ ) but there is no appropriate definition of such a supermodule. To overcome this obstacle, we construct to any normal supersubgroup some biggest normal supersubgroup over which the quotient is affine. Next step is to prove that the original supersubgroup coincides with this new one. For $\operatorname{char} K=p>0$ we use some trick with a Frobenius map. The characteristic zero case is much more complicated and we have to introduce a notion of a pseudoconnected component of a supergroup to reduce our problem to the case of a finite normal supersubgroup. Besides, we use induction on superdimensions of Lie superalgebras of our supergroups and some properties of adjoint representations. In the final section an example of faithfully exact supersubgroup is given. 
This is a Levi supersubgroup of a general linear supergroup. Furthermore, we construct an affine superscheme $X$ on which a finite (odd) unipotent supergroup $G$ acts in a such way, that neither $X \tilde{\tilde{\mu}} G$, nor $X \tilde{/} G$ is affine.

In seventh and eighth section a partial answer for the following Brundan's question is given. Let $G$ be an algebraic supergroups and $H$ be its supersubgroup such that $H_{e v}$ is reductive. Is it true that $G \tilde{\tilde{J}} H$ is affine? We show that the answer is positive if char $K=$ $p>0$ or $G$ is finite.

\section{Super(co)algebras and super(co)modules}

We follow definitions and notations from [1, 2] (see also [8]). Let $K$ be a field of characteristic $p \neq 2$. For a $K$-vector superspace $V$ its superdimension is defined as $\operatorname{sim} V=$ ( $\left.\operatorname{dim} V_{0}, \operatorname{dim} V_{1}\right)$. Let $A$ be a (associative) superalgebra over $K$. Denote by $A-\operatorname{smod}$ (or smod-A, respectively) the category of all left (or right, respectively) $A$-supermodules with even morphisms. Let $X$ be a left (or right) $A$-supermodule with $\mathbf{Z}_{2}$-grading $X=X_{0} \oplus X_{1}$. For any $x \in X$ denote by $x_{0}$ and $x_{1}$ its homogeneous components, that is $x_{0} \in X_{0}, x_{1} \in X_{1}$ and $x=x_{0}+x_{1}$. If $X \in \operatorname{smod}-A, Y \in A-\operatorname{smod}$, then the tensor product $X \otimes_{A} Y$ has a natural $\mathbf{Z}_{2}$-grading given by $|x \otimes y|=|x|+|y|(\bmod 2)$ for $x \in X, y \in Y$.

A superalgebra $A$ has the opposite companion $A^{\circ}$ whose underlying superspace coincides with $A$ and the multiplication in $A^{\circ}$ is defined by $a * b=(-1)^{|a||b|} b a$. It is clear that $\left(A^{\circ}\right)^{\circ}=A$. We have an equivalence of categories $A-\operatorname{smod} \simeq \operatorname{smod}-A^{\circ}$ given by $M \mapsto M^{\circ}$ for $M \in A-\operatorname{smod}$, where $M^{\circ}$ coincides with $M$ as a superspace and a structure of a right supermodule on $M^{\circ}$ is defined by $m * a=(-1)^{|m||a|}$ am for $a \in A, m \in M$. For any $X \in \operatorname{smod}-A, Y \in A-\operatorname{smod}$, we have an isomorphism $X \otimes_{A} Y \simeq Y^{\circ} \otimes_{A^{\circ}} X^{\circ}$ given by $x \otimes y \mapsto(-1)^{|x||y|} y \otimes x$ for $x \in X, y \in Y$ which is functorial in $X$ and $Y$.

A superalgebra $A$ is called commutative if any homogeneous elements $a, b \in A$ satisfy $a b=(-1)^{|a||b|} b a$. In particular, $A=A^{\circ}$ and $A-\operatorname{smod} \simeq \operatorname{smod}-A$. In other words, any one-sided $A$-supermodule has a canonical structure of a $A$-superbimodule. Denote by $S A g_{K}$ the category of all commutative $K$-superalgebras with even morphisms. If $\phi: B \rightarrow A, \psi: B \rightarrow C$ are morphisms in $S A l g_{K}$, then $A \otimes_{B} C \in S A l g_{K}$ (see [8]). From now on, all superalgebras are commutative unless otherwise stated.

Let $K[m \mid n]=K\left[t_{1}, \ldots, t_{m} \mid z_{1}, \ldots z_{n}\right]$ be a free commutative superalgebra with free generators $t_{1}, \ldots, t_{m}, z_{1}, \ldots z_{n}$, where $\left|t_{i}\right|=0,\left|z_{j}\right|=1$ for $1 \leq i \leq m, 1 \leq j \leq n$. It can be identified with the symmetric superalgebra $S(V)$ of a superspace $V$, where $\operatorname{dim} V_{0}=$ $m, \operatorname{dim} V_{1}=n$ (see [1]).

Lemma 1.1 Let $R=R_{0} \bigoplus R_{1}$ be a superalgebra. Then

i) If $R$ is finitely generated, then $R$ is noetherian;

ii) Every one-sided superideal of $R$ is two-sided;

iii) A prime ideal $\mathcal{P}$ of $R$ has a form $\mathcal{P}=\mathcal{P}_{0}+R_{1}$, where $\mathcal{P}_{0}$ is a prime ideal of $R_{0}$. If $\mathcal{M}$ is a maximal one-sided ideal of $R$, then $\mathcal{M}=\mathcal{M}_{0}+R_{1}$, where $\mathcal{M}_{0}$ is a maximal ideal of $R_{0}$. In particular, all these ideals are superideals.

Proof. The statement ii) is obvious. To prove i) we notice that $K[m \mid n]$ is finitely generated module over $K\left[t_{1}, \ldots, t_{m}\right]$. Finally, iii) holds since $R R_{1}$ is a nil ideal. 
Remark 1.1 It is not true that every one-sided ideal in a superalgebra is necessary twosided. For example, the left ideal $K[1 \mid 2]\left(t_{1}+z_{1}\right)$ is not two-sided.

Denote by $\sqrt[R]{I}$ the prime radical of a superideal $I$. It is the intersection of all prime ideals containing $I$. It is not difficult to see that

$$
\sqrt[R]{I}=\left\{r \in R \mid \exists n, r^{n} \in I\right\}=\left\{r \in R \mid \exists n, r_{0}^{n} \in I_{0}\right\},
$$

where for the last equality we used the obvious formula $r^{n}=r_{0}^{n}+n r_{0}^{n-1} r_{1}$.

Let $A$ be a (not necessary commutative) superalgebra. A left supermodule $Y \in A-$ smod (a right supermodule $Y \in \operatorname{smod}-A$, respectively) is called flat if the functor $X \rightarrow X \otimes_{A} Y\left(X \rightarrow Y \otimes_{A} X\right.$, respectively $)$ is an exact functor from the category smod $-A$ ( $A-$ smod, respectively) to the category of superspaces.

Next, $Y \in A-\operatorname{smod}(Y \in \operatorname{smod}-A$, respectively) is called faithfully flat if the corresponding functor is faithfully exact, that is the exactness of any sequence $X^{\prime} \rightarrow X \rightarrow$ $X^{\prime \prime}$ in smod $-A$ (in $A-$ smod, respectively) is equivalent to the exactness of the sequence of superspaces $X^{\prime} \otimes_{A} Y \rightarrow X \otimes_{A} Y \rightarrow X^{\prime \prime} \otimes_{A} Y\left(Y \otimes_{A} X^{\prime} \rightarrow Y \otimes_{A} X \rightarrow Y \otimes_{A} X^{\prime \prime}\right.$, respectively). Using the previous discussion, a left $A$-supermodule $Y$ is flat (faithfully flat) iff the right $A^{\circ}$-supermodule $Y^{\circ}$ is flat (faithfully flat). If $A$ is commutative, then any supermodule is left flat (left faithfully flat) iff it is right flat (right faithfully flat). Most of standard characterizations of flatness or faithful flatness from [3] can be easily translated to the supercase. We call such translation a superversion of the corresponding statement. Proofs of superversions of results from [3] that are not difficult are left to the reader.

Let $A$ be an algebra and let $S$ be a multiplicative set belonging to the center of $A$. The algebra of fractions $S^{-1} A$ and the left (or right, respectively) $S^{-1} A$-module of fractions $S^{-1} X \simeq S^{-1} A \otimes_{A} X$ (or $S^{-1} X \simeq X \otimes_{A} S^{-1} A$, respectively) for left (or right, respectively) $A$-module $X$ is defined in the usual way. If $A$ is a superalgebra, $X$ is an $A$-supermodule and $S \subseteq A_{0}$, then $S^{-1} X$ is also an $A$-supermodule with $\mathbf{Z}_{2}$-grading given by $\left(S^{-1} X\right)_{i}=S^{-1} X_{i}$ for $i=0,1$.

Lemma 1.2 Let $A$ and $S$ be as above. Then

i) $S^{-1} A$ is a flat $A$-module;

ii) If central elements $a_{1}, \ldots, a_{n} \in A$ generate $A$ as an ideal, then the algebra $\prod_{1 \leq i \leq n} A_{a_{i}}$ is a faithfully flat (left and right) A-module.

Proof. The first statement is an easy generalization of Theorem 1 from [3], II, §2. To prove the second statement, use Proposition 1 from [3, I, $\S 3$ and observe that all powers of the elements $a_{1}, \ldots, a_{n}$ again generate $A$ as an ideal.

Lemma 1.3 A superalgebra $A$ is generated by elements $a_{1}, \ldots, a_{n}$ as a left (or right) ideal iff $A$ is generated by their even components.

Proof. Assume that $1=\sum_{1 \leq i \leq n} b_{i} a_{i}$. Set $a_{i, k}, b_{i, k} \in A_{k}$, for $1 \leq i \leq n$ and $k=0,1$. Then

$$
\sum_{1 \leq i \leq n} b_{i, 0} a_{i, 0}=1-\sum_{1 \leq i \leq n} b_{i, 1} a_{i, 1}
$$

and the element $1-\sum_{1 \leq i \leq n} b_{i, 1} a_{i, 1} \in 1+A A_{1}$ is invertible. 
Corollary 1.1 If $A$ is a superalgebra and $a_{1}, \ldots, a_{n} \in A_{0}$ generate $A_{0}$ as ideal, then the superalgebra $\prod_{1 \leq i \leq n} A_{a_{i}}$ is a faithfully flat (left and right) A-module.

The spectrum of all maximal ideals of a superalgebra $A$ is denoted by $\operatorname{Max}(A)$. For any $\mathcal{M} \in \operatorname{Max}(A)$ we denote by $N_{\mathcal{M}}$ an even localization of an $A$-supermodule $N$. More precisely, $N_{\mathcal{M}}=\left(A_{0} \backslash \mathcal{M}_{0}\right)^{-1} N$.

In what follows all algebras are superalgebras.

Lemma 1.4 If $\mathcal{M} \in \operatorname{Max}(A)$, then the algebra $A_{\mathcal{M}}$ is local and $\mathcal{M} A_{\mathcal{M}}$ is its Jacobson radical. In particular, the left (and right) $A$-module $B=\bigoplus_{\mathcal{M} \in \operatorname{Max}(A)} A_{\mathcal{M}}$ is faithfully flat.

Proof. If an element $a=a_{0}+a_{1} \in A$ is such that $a_{0} \notin \mathcal{M}_{0}$, then $a^{-1}=\frac{1}{a_{0}}-\frac{a_{1}}{a_{0}^{2}} \in A_{\mathcal{M}}$. Next, by Lemma 1.2, the $A$-module $B$ is flat and $\mathcal{M} B \neq B, B \mathcal{M} \neq B$ for all $\mathcal{M} \in \operatorname{Max}(A)$.

Lemma 1.5 A morphism of left (or right) A-modules $M \rightarrow N$ is a monomorphism (an epimorphism or an isomorphism, respectively) iff the induced morphism $M_{\mathcal{M}} \rightarrow N_{\mathcal{M}}$ is injective (surjective or bijective, respectively) for any $\mathcal{M} \in \operatorname{Max}(A)$.

Proof. A word-by-word repetition of the proof of Theorem 1, II, §3, 3] combined with Lemma 1.4.

Lemma 1.6 A left (or right) A-module $M$ is flat (faithfully flat, respectively) iff $M_{\mathcal{M}}$ is a flat (faithfully flat, respectively) $A_{\mathcal{M}}$-module for all $\mathcal{M} \in \operatorname{Max}(A)$.

Proof. The necessary condition is a consequence of Proposition 8, I, $\S 2$ and Proposition 4, I, $\S 3$ from [3]. For the sufficient condition, assume that $M_{\mathcal{M}}$ is a flat (and, for example, right) $A$-module for all $\mathcal{M} \in \operatorname{Max}(A)$. If $N_{1} \rightarrow N_{2}$ is an inclusion of left $A$-modules, combine $\left(M \otimes_{A} N\right)_{\mathcal{M}} \simeq M_{\mathcal{M}} \otimes_{A} N$ with Lemma 1.5 to obtain that $M \otimes_{A} N_{1} \rightarrow M \otimes_{A} N_{2}$ is again an inclusion. Additionally, if $M_{\mathcal{M}}$ is faithfully flat for all $\mathcal{M} \in \operatorname{Max}(A)$, then $\mathcal{M} M_{\mathcal{M}} \neq M_{\mathcal{M}}$ implies $\mathcal{M} M \neq M$.

Proposition 1.1 Let $\phi: A \rightarrow B$ be a morphism of superalgebras and let $M$ be a $B-$ $A$-bimodule (an $A-B$-bimodule, respectively) such that $m a=\phi(a) m$ for $m \in M, a \in$ $A_{0}$ (am $=m \phi(a)$ for $m \in M, a \in A_{0}$, respectively). Then the following properties are equivalent:

i) $M$ is a flat $A$-module;

ii) $M_{\mathcal{N}}$ is a flat $A$-module for all $\mathcal{N} \in \operatorname{Max}(B)$;

iii) $M_{\mathcal{N}}$ is a flat $A_{\mathcal{M}}$-module for every $\mathcal{N} \in \operatorname{Max}(B)$, where $\mathcal{M}=\phi^{-1}(\mathcal{N})$.

Proof. Use Lemmas 1.2 and 1.5 to copy the proof of Proposition 15, II, §3, [3].

Corollary 1.2 If $M$ is a B-supermodule, then the conditions of Proposition 1.1 hold automatically. In particular, the properties i)-iii) are equivalent.

Let $M$ be a flat left (or right, respectively) $A$-module over an algebra $A$ and let $\alpha$ be an automorphism of $A$. Denote by $M^{\alpha}$ an $A$-module such that $M^{\alpha}=M$ and $a \star m=\alpha(a) m$ (or $m \star a=m \alpha(a)$, respectively) for $a \in A, m \in M$. The corollary after Proposition 13, I, $\S 2$ of [3] implies that $M$ is a flat $A$-module iff $M^{\alpha}$ is a flat $A$-module. 
All necessary definitions and notations concerning supercoalgebras, Hopf superalgebras and supercomodules over them can be found in [1, 2]. If $C$ is a supercoalgebra and $V$ is a left (or right, respectively) $C$-supercomodule, then a counit of $C$ and a coaction map $V \rightarrow C \otimes V$ (or $V \rightarrow V \otimes C$, respectively) are denoted by $\epsilon_{C}$ and $\tau_{V}$ correspondingly. A comultiplication of $C$ is denoted by $\delta_{C}$. Additionally, if $C$ is a Hopf superalgebra, then its antipode is denoted by $s_{C}$. The category of left (or right, respectively) $C$-supercomodules with even morphisms is denoted by $C-\operatorname{scomod}$ (or scomod $-C$, respectively). If $V \in$ scomod $-C, W \in C-$ scomod, then one can define a cotensor product

$$
V \square_{C} W=\left\{x \in V \otimes W \mid\left(\tau_{V} \otimes i d_{W}\right)(x)=\left(i d_{V} \otimes \tau_{W}\right)(x)\right\} .
$$

A left (or right, respectively) $C$-supercomodule $V$ is called (faithfully) coflat if the functor $W \rightarrow W \square_{C} V$ (or $W \rightarrow V \square_{C} W$, respectively) is (faithfully) exact, where $W \in \operatorname{scomod}-C$ (or $W \in C-$ scomod, respectively).

Lemma 1.7 (see A.2.1, [14]) A right (or left) $C$-supercomodule $V$ is coflat (faithfully coflat, respectively) iff $V$ is injective (an injective cogenerator, respectively).

Proof. Let $V \in \operatorname{scomod}-C, W \in C-$ scomod and $\operatorname{dim} W<\infty$. The dual superspace $W^{*}$ has a uniquely defined structure of a right $C$-supercomodule given by $\sum f_{1}(w) c_{2}^{\prime}=$ $\sum f\left(w_{1}\right) c_{2}$, where $\tau_{W}(w)=\sum c_{2} \otimes w_{1}, \tau_{W^{*}}(f)=\sum f_{1} \otimes c_{2}^{\prime}$ for $w \in W, f \in W^{*}$. We have an isomorphism of superspaces $V \square_{C} W \rightarrow H_{C} m_{C}\left(W^{*}, V\right)$ induced by $v \otimes w(f)=f(w) v$ for $v \in V, w \in W, f \in W^{*}$. Since this isomorphism is functorial in $W$, we conclude the proof as in [14.

\section{$2 \quad K$-functors and $K$-sheafs (faisceaux)}

Following the book [4] we call a functor from the category $S A l g_{K}$ to the category of sets a $K$-functor. The category of all $K$-functors is denoted by $\mathcal{F}$. A $K$-functor $S S p R$ defined as $\operatorname{SSp} R(A)=\operatorname{Hom}_{S A l g_{K}}(R, A)$ for $A \in S A l g_{K}$ is called an affine superscheme (this definition is different from the definition used in [1] since we do not suppose that $R$ is finitely generated). The superalgebra $R \in S A l g_{K}$ is called a coordinate superalgebra of the superscheme $S S p R$. If $X=S S p R$, then $R$ is also denoted by $K[X]$.

Lemma 2.1 (Yoneda's lemma, 4], part I, (1.3)) For an affine superscheme SSp $R$ and a $K$-functor $X$ there is a canonical isomorphism $\operatorname{Mor}(S S p R, X) \simeq X(R)$ which is functorial in both arguments. In particular, the category $S A l g_{K}$ is anti-equivalent to the full subcategory of affine superschemes.

Proof. The statement of the lemma is a partial case of more general theorem about covariant representable functors (see [5], Theorem 1.6). The isomorphism is given by $f \mapsto x_{f}=f(R)\left(i d_{R}\right)$ for $f \in \operatorname{Mor}(S S p R, X)$ and the inverse map is given by $x \mapsto f_{x}$, where $f_{x}(\alpha)=X(\alpha)(x)$ for $x \in X(R), \alpha \in \operatorname{Hom}_{S A l g_{K}}(R, A)$ and $A \in S A l g_{K}$.

Corollary 2.1 The universal property of $A \otimes_{B} C$ implies a canonical isomorphism

$$
S S p A \times_{S S p} B S S p C \simeq S S p A \otimes_{B} C .
$$


The affine superscheme $\mathbf{A}^{m \mid n}=S S p K\left[t_{1}, \ldots, t_{m} \mid z_{1}, \ldots z_{n}\right]$ is called $(m \mid n)$-affine superspace. It is clear that $\mathbf{A}^{m \mid n}(B)=B_{0}^{m} \bigoplus B_{1}^{n}$ for $B \in S A l g_{K}$. In particular, $\mathbf{A}^{1 \mid 1}(B)=B$ for any superalgebra $B$.

Let $I$ be a superideal of $R \in S A l g_{K}$. Denote by $V(I)$ a closed subfunctor of $S S p R$ corresponding to $I$. By definition, $V(I)(A)=\{\phi \in S S p R(A) \mid \phi(I)=0\}$. It is obvious that $V(I) \simeq S S p R / I$. All standard properties of closed subfunctors of affine schemes mentioned in [4], part I, (1.4) are translated to the category of affine superschemes per verbatim.

Let $X$ be an affine superscheme. A functor $Y \subseteq X$ is called open if

$$
Y(A)=\left\{x \in X(A) \mid \sum_{f \in I} A x(f)=A\right\}
$$

for a subset $I \subseteq K[X]$ and $A \in S A l g_{K}$. Denote this functor by $D(I)$.

Lemma 2.2 i) If $J$ is the smallest superideal containing $I$, then $D(I)=D(J)=D\left(J_{0}\right)$; ii) Let $I$ and $I^{\prime}$ be superideals of $R$. Then $D(I) \subseteq D\left(I^{\prime}\right)$ iff $\sqrt[R]{I} \subseteq \sqrt[R]{I^{\prime}}$. Additionally, $D(I)=D(\sqrt[R]{I})=D\left(\sqrt[R_{0}]{I_{0}}\right)$.

Proof. All statements can be proved by the same trick with a representation of unit as in Lemma 1.3 and by the standard reductions to quotients modulo prime ideals (see [4], part I (1.6)).

An important example of an open subfunctor is a so-called principal open subfuctor $X_{f}=D(\{f\})$ for $f \in K[X]$. It can be checked easily that $X_{f}=X_{f_{0}}$ is again an affine superscheme and $K\left[X_{f}\right]=K[X]_{f_{0}}$. All other properties of open subfunctors mentioned in [4], part I (1.6) are easily translated to the category of affine superschemes.

Let $G$ be a group $K$-functor, that is a $K$-functor to the category of groups. We say that $G$ acts on a $K$-functor $X$ on the right (on the left, respectively) if there is a morphism of functors $f: X \times G \rightarrow X(g: G \times X \rightarrow X$, respectively) such that $f\left(i d_{X} \times \mu\right)=f\left(f \times i d_{G}\right)$ and $f i_{E}=i d_{X}\left(g\left(\mu \times i d_{X}\right)=g\left(i d_{G} \times g\right)\right.$ and $g j_{E}=i d_{X}$, respectively). Here $\mu: G \times G \rightarrow G$ is a multiplication of $G$ and $i_{X}: X \rightarrow X \times G$ is defined as $i_{X}(R)(x)=\left(x, 1_{G(R)}\right)\left(j_{X}(R)(x)=\left(1_{G(R)}, x\right)\right.$, respectively) for $x \in X(R), R \in S A l g_{K}$. From now on we consider any action on right unless otherwise stated.

It is obvious that the category of affine group superschemes (= affine supergroups) is anti-equivalent to the category of commutative Hopf superalgebras (see [1, 4]). If $G$ is an affine supergroup, then denote by $\epsilon_{G}, \delta_{G}$ and $s_{G}$ the counit, comultiplication and antipode of $K[G]$ correspondingly. If $K[G]$ is finitely generated, then $G$ is called an algebraic supergroup. Closed supersubgroups $H \leq G$ are in one-to-one correspondence with Hopf superideals $I_{H} \subseteq K[G]$ such that $H=V\left(I_{H}\right)$. If $X$ and $G$ are affine, then an action of $G$ on $X$ is uniquely defined by a morphism of superalgebras $\tau: K[X] \rightarrow K[X] \otimes K[G]$ with respect to which $K[X]$ is a right $K[G]$-supercomodule.

Let $V$ be a vector superspace of superdimension $(m, n)$. Denote by $G L(V)$ or by $G L(m \mid n)$ the corresponding general linear supergroup. More precisely, $G L(V)$ is a group $K$-functor such that for any $B \in S A l g_{K}$ the group $G L(V)(B)$ consists of all even and $B$-linear automorphisms of $V \otimes B$. It is not difficult to see that $G L(V)$ is an algebraic supergroup (see [1, 24, 25] for more details). A linear representation of a group $K$-functor $G$ is a morphism of group $K$-functors $\rho: G \rightarrow G L(V)$. In this case the superspace $V$ 
is called $G$-supermodule. If $G$ is an affine supergroup, then $V$ is a $G$-supermodule iff it is a right $K[G]$-supercomodule (see [1, 4]). In fact, fix a basis $v_{1}, \ldots, v_{m+n}$ of $V$ such that $\left|v_{i}\right|=0$ if $1 \leq i \leq m$ and $\left|v_{i}\right|=1$ otherwise. Set $\tau_{V}\left(v_{i}\right)=\sum_{1 \leq j \leq m+n} v_{j} \otimes r_{j i}$ for $1 \leq i \leq m+n$. Then $\rho(g)=\left(g\left(r_{i j}\right)\right)$ for $g \in G(B)$ and $B \in S A l g_{K}$.

From now on, any group $K$-functor is affine unless otherwise stated. Let $W$ be a supersubspace of a finitely dimensional $G$-supermodule $V$. The stabilizer $\operatorname{Stab}_{G}(W)$ is a group subfunctor defined as $\operatorname{Stab}_{G}(W)(A)=\{g \in G(A) \mid g(W \otimes 1) \subseteq W \otimes A\}$ for $A \in S A l g_{K}$. It is easy to see that $\operatorname{Stab}_{G}(W)$ is a closed supersubgroup of $G$. In fact, without loss of generality one can assume that $v_{1}, \ldots, v_{s}, v_{m+1}, \ldots, v_{m+t}$ for $s \leq m, t \leq n$ is a basis of $W$. Denote by $M$ the set of indexes $\{1, \ldots, s, m+1, \ldots m+t\}$. Then $g \in \operatorname{Stab}_{G}(W)(A)$ iff $g\left(r_{j i}\right)=0$ for all $j \notin M, i \in M$ and $A \in S A l g_{K}$.

Let $R_{1}, \ldots, R_{n}$ be a finite family of commutative $R$-superalgebras with respect to a set of morphisms $\iota_{R}^{R_{i}}: R \rightarrow R_{i}$ in $S$ Alg $g_{K}$. Such a family is called faithfully flat covering of $R$ (ff-covering, for short) whenever $R$-supermodule $R_{1} \times \ldots \times R_{n}$ is faithfully flat. We say that $R$-superalgebra $R^{\prime}$ is finitely presented if $R^{\prime} \simeq R[m \mid n] / I$, where $R[m \mid n]=R \otimes K[m \mid n]$ and $I \subseteq R[m \mid n]$ is a finitely generated superideal. It is not difficult to check that $R^{\prime}$ is a finitely presented $R$-superalgebra iff $R^{\prime} \simeq R \otimes_{A} A[m \mid n] / I$, where $A$ is a finitely generated supersubalgebra of $R$ (see [6], I, §3). Following [4] we call a ff-covering $R_{1}, \ldots, R_{n} f p p f$ covering if all $R_{i}$ are finitely presented $R$-superalgebras.

A $K$-functor $X$ is called dur $K$-sheaf (or faisceau dur) if for any ff-covering $R_{1}, \ldots R_{n}$ of a superalgebra $R$ the diagram

$$
X(R) \rightarrow \prod_{1 \leq i \leq n} X\left(R_{i}\right) \rightarrow \prod_{1 \leq i, j \leq n} X\left(R_{i} \otimes_{R} R_{j}\right)
$$

is exact, where the last two maps are induced by morphisms $R_{i} \rightarrow R_{i} \otimes_{R} R_{j}$ and $R_{i} \rightarrow R_{j} \otimes_{R} R_{i}$, respectively, defined as $a \mapsto a \otimes 1$ and $b \mapsto 1 \otimes b$ for $a, b \in R_{i}$. This property is equivalent to the following two conditions. For all $R_{1}, \ldots, R_{n}, R, R^{\prime} \in S$ Alg $_{K}$, where $R^{\prime}$ is a faithfully flat $R$-supermodule, there is a canonical bijection $X\left(\prod_{1 \leq i \leq n} R_{i}\right) \simeq$ $\prod_{1 \leq i \leq n} X\left(R_{i}\right)$ and the diagram

$$
X(R) \rightarrow X\left(R^{\prime}\right) \underset{\rightarrow}{\rightarrow} X\left(R^{\prime} \otimes_{R} R^{\prime}\right)
$$

is exact, see [4]. Replacing ff-coverings by fppf-coverings we obtain a definition of a $K$ sheaf (or faisceau), cf. [6, 4]. Denote the full subcategory of $K$-sheafs (dur $K$-sheafs,

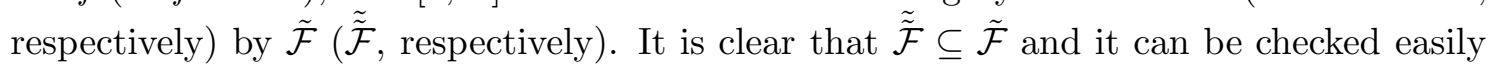
that any affine supersheme is a dur $K$-sheaf, see [4], part I (5.3).

For a $K$-functor $X$ one can construct an associated $K$-sheaf $\tilde{X}$ and a dur $K$-sheaf $\tilde{X}$ following the way described in [6], III, $\S 1$. We consider a partial case following [4], part I (5.4). Assume that the $K$-functor $X$ satisfies the following conditions :

$\left({ }^{*}\right) X\left(\prod_{1<i<n} R_{i}\right) \simeq \prod_{1<i<n} X\left(R_{i}\right)$ for all $R_{1}, \ldots, R_{n} \in S A l g_{K}$;

(**) $X(R) \rightarrow X\left(R^{\prime}\right)$ is an inclusion for arbitrary fppf-covering $R^{\prime}$ of superalgebra $R$.

The family of all $K$-functors satisfying the properties $\left(^{*}\right)$ and $(* *)$ is closed under direct products. Define a partial order on the set of all fppf-coverings of a superalgebra $R$ by the following rule: $R^{\prime} \leq R^{\prime \prime}$ if $R^{\prime \prime}$ is a fppf-covering of $R^{\prime}$. Clearly, this poset is directed since 
$R^{\prime}, R^{\prime \prime} \leq R^{\prime} \otimes_{R} R^{\prime \prime}$ for any two fppf-coverings of $R$. For each superalgebra $R$ define the direct spectrum

$$
\mathcal{X}(R)=\left\{X\left(R^{\prime}, R\right)=\operatorname{ker}\left(X\left(R^{\prime}\right) \rightarrow X\left(R^{\prime} \otimes_{R} R^{\prime}\right)\right) \mid R^{\prime} \text { is a fppf-covering of } R\right\}
$$

with canonical inclusions $X\left(R^{\prime}, R\right) \rightarrow X\left(R^{\prime \prime}, R\right)$ for any couple $R^{\prime} \leq R^{\prime \prime}$. Observe that $R^{\prime \prime} \otimes_{R} R^{\prime \prime}$ is a fppf-covering of $R^{\prime} \otimes_{R} R^{\prime}$ and set $\tilde{X}(R)=\lim _{\rightarrow} \mathcal{X}(R)$. The functor $\tilde{X}$ is the required completion of $X$ with respect to the Grothendieck topology of fppf-coverings. A canonical inclusion $\alpha_{X}: X \rightarrow \tilde{X}$ induces a canonical bijection $\operatorname{Mor}(\tilde{X}, Y) \rightarrow \operatorname{Mor}(X, Y)$ for any $K$-sheaf $Y$. One gets easily that if $X$ is a subfunctor of a $K$-sheaf $Y$ satisfying $(*)$, then $\tilde{X} \subseteq Y$. Moreover,

$$
\tilde{X}(R)=\left\{y \in Y(R) \mid \text { there is } R^{\prime} \geq R \text { such that } Y\left(\iota_{R}^{R^{\prime}}\right)(y) \in X\left(R^{\prime}\right)\right\} .
$$

To prove all of the above statements we only need a superversion of Proposition 4 [3], I, $\S 3$ together with an additional statement which says that $R=\prod_{1 \leq i \leq n} R_{i} \leq R^{\prime}$ iff $R_{i}=R e_{i} \leq R^{\prime} e_{i}$ for any $i$, where $e_{i}=(0, \ldots, \quad \underbrace{1}, \ldots, 0)$ and $1 \leq i \leq n$. Arguments $i$-th place

for dur $K$-sheafs are the same, except that fppf-coverings are replaced by ff-coverings.

Lemma 2.3 Let $G$ be a group $K$-functor that satisfies $\left(^{*}\right)$ and $\left({ }^{* *}\right)$ for all ff-coverings (fppf-coverings, respectively). Then $\tilde{\tilde{G}}(\tilde{G}$, respectively) is again a group dur K-sheaf (a group $K$-sheaf, respectively) and the canonical inclusion $G \rightarrow \tilde{\tilde{G}}(G \rightarrow \tilde{G}$, respectively) is a morphism of group functors.

Proof. Let $g, g_{1}, g_{2} \in \tilde{\tilde{G}}(A)$. For a suitable ff-covering $B$ of a superalgebra $A$ we set $\bar{g}=\tilde{\tilde{G}}\left(\iota_{A}^{B}\right)(g)$ and $\bar{g}_{i}=\tilde{\tilde{G}}\left(\iota_{A}^{B}\right)\left(g_{i}\right) \in G(B)$ for $i=1,2$. The pair $\left(\bar{g}_{1}, \bar{g}_{2}\right)$ belongs to $(G \times G)(B, A)$ which implies $\bar{g}_{1} \bar{g}_{2} \in G(B, A)$. Define $g_{1} g_{2}=\tilde{\tilde{G}}\left(\iota_{A}^{B}\right)^{-1}\left(\bar{g}_{1} \bar{g}_{2}\right) \in \tilde{\tilde{G}}(A)$ and $g^{-1}=\tilde{\tilde{G}}\left(\iota_{A}^{B}\right)^{-1}\left(\bar{g}^{-1}\right)$. These definitions do not depend on the choice of $B$. If $\phi: A \rightarrow C$ is a morphism in $S A \lg _{K}, B$ and $B^{\prime}$ are ff-coverings of $A$ and $C$, respectively, then $B \otimes_{A} B^{\prime}$ is a ff-covering of $C$ and $B^{\prime}$, respectively. Denote by $\alpha$ and $\beta$ the morphisms $B \rightarrow B \otimes_{A} B^{\prime}$ and $B^{\prime} \rightarrow B \otimes_{A} B^{\prime}$ given by $\alpha(b)=b \otimes 1, \beta\left(b^{\prime}\right)=1 \otimes b^{\prime}$ for $b \in B, b^{\prime} \in B^{\prime}$ and set $x_{i}=\tilde{\tilde{G}}(\phi)\left(g_{i}\right), \bar{x}_{i}=\tilde{\tilde{G}}\left(\iota_{C}^{B^{\prime}}\right)\left(x_{i}\right)$ for $i=1,2$. Then

$\tilde{\tilde{G}}\left(\beta \iota_{C}^{B^{\prime}} \phi\right)\left(g_{1} g_{2}\right)=\tilde{\tilde{G}}\left(\alpha \iota_{A}^{B}\right)\left(g_{1} g_{2}\right)=G(\alpha)\left(\bar{g}_{1}\right) G(\alpha)\left(\bar{g}_{2}\right)=G(\beta)\left(\bar{x}_{1}\right) G(\beta)\left(\bar{x}_{2}\right)=\tilde{\tilde{G}}\left(\beta \iota_{C}^{B^{\prime}}\right)\left(x_{1} x_{2}\right)$

and since the map $\tilde{\tilde{G}}\left(\beta \iota_{C}^{B^{\prime}}\right)$ is injective we infer that $\tilde{\tilde{G}}(\phi)\left(g_{1} g_{2}\right)=x_{1} x_{2}$. The remaining statements of the lemma are now obvious. The case of $K$-sheafs is anologous.

\section{Superalgebras of distributions and Lie superalgebras}

Let $X$ be an affine superscheme. Following [4] we call any element of $\operatorname{Dist}_{n}(X, \mathcal{M})=$ $\left(K[X] / \mathcal{M}^{n+1}\right)^{*}$ a distribution on $X$ with support at $\mathcal{M} \in \operatorname{Max}(K[X])$ of orded $\leq n$ (notice that $\operatorname{Max}(K[X])$ is obviously identified with $X(K))$. We have $\bigcup_{n \geq 0} \operatorname{Dist}_{n}(X, \mathcal{M})=$ $\operatorname{Dist}(X, \mathcal{M}) \subseteq K[X]^{*}$. If $g: X \rightarrow Y$ is a morphism of affine superschemes, then it 
induces a morphism of filtered superspaces $d g_{\mathcal{M}}: \operatorname{Dist}(X, \mathcal{M}) \rightarrow \operatorname{Dist}\left(Y,\left(g^{*}\right)^{-1}(\mathcal{M})\right)$. In particular, if $X=V(I)$ is a closed supersubscheme of $Y$, then $\operatorname{Dist}(X, \mathcal{M})$ is identified with a filtered supersubspace $\{\phi \in \operatorname{Dist}(Y, \mathcal{M}) \mid \phi(I)=0\}$, where $I \subseteq \mathcal{M}$.

If $X$ is an algebraic supergroup and $\mathcal{M}=\operatorname{ker} \epsilon_{X}$, then $\operatorname{Dist}(X, \mathcal{M})$ is denoted by $\operatorname{Dist}(X)$. In this case $\operatorname{Dist}(X)$ has a structure of a Hopf superalgebra with a multiplication $\phi \psi(f)=\sum(-1)^{|\psi|\left|f_{1}\right|} \phi\left(f_{1}\right) \psi\left(f_{2}\right)$ for $\phi, \psi \in \operatorname{Dist}(X), f \in K[X]$ and $\delta_{X}(f)=\sum f_{1} \otimes f_{2}$, with a unit $\epsilon_{X}$ and with a counit $\epsilon_{\operatorname{Dist}(X)}: \phi \mapsto \phi(1)$. The comultiplication of $\operatorname{Dist}(X)$ is dual to the multiplication of $K[X]$, cf. [24, 25]. Finally, an antipode $s_{\operatorname{Dist}(X)}$ is defined by $s_{\operatorname{Dist}(X)}(\phi)(f)=\phi\left(s_{X}(f)\right)$ for $\phi \in \operatorname{Dist}(X)$ and $f \in K[X]$.

We have $\operatorname{Dist}_{k}(X) \operatorname{Dist}_{l}(X) \subseteq \operatorname{Dist}_{k+l}(X)$ for all $k, l \geq 0$, that is the superalgebra $\operatorname{Dist}(X)$ is a filtered algebra. The superspace $\operatorname{Lie}(X)=\left\{\phi \in \operatorname{Dist}_{1}(X) \mid \phi(1)=0\right\}$ has a Lie superalgebra structure given by $[\phi, \psi]=\phi \psi-(-1)^{|\phi||\psi|} \psi \phi$. As a Hopf superalgebra, $\operatorname{Dist}(X)$ is cocommutative which means that $\delta_{\operatorname{Dist}(X)}(\phi)=\sum \phi_{1} \otimes \phi_{2}=\sum(-1)^{\left|\phi_{1}\right|\left|\phi_{2}\right|} \phi_{2} \otimes$ $\phi_{1}$. Additionally, each $\operatorname{Dist}_{n}(X)$ is a supersubcoalgebra of $\operatorname{Dist}(X)$. For arbitrary morphism of algebraic supergroups $g: X \rightarrow Y$ its differential $d g=d g_{\mathcal{M}}: \operatorname{Dist}(X) \rightarrow \operatorname{Dist}(Y)$ is a homomorphism of filtered Hopf superalgebras. In particular, its restriction to $\operatorname{Lie}(X)$ is a homomorphism of Lie superalgebras.

Let $L$ be a Lie superalgebra. Denote by $U(L)$ its (universal) enveloping superalgebra, see [26]. The superalgebra $U(L)$ is a Hopf superalgebra with a comultiplication defined by $\delta_{U(L)}(x)=x \otimes 1+1 \otimes x$ for $x \in L$. Its counit $\epsilon_{U(L)}$ is defined by $\epsilon_{U(L)}(L)=0$ and its antipode $s_{U(L)}$ is defined by $s_{U(L)}(x)=-x$ for $x \in L$. The antipode $s_{U(L)}$ is an antiautomorphism of $U(L)$ such that $s_{U(L)}(u v)=(-1)^{|u||v|} s_{U(L)}(v) s_{U(L)}(u)$ for $u, v \in U(L)$. The Hopf superalgebra $U(L)$ is obviously filtered and cocommutative.

The inclusion $\operatorname{Lie}(X) \subseteq \operatorname{Dist}(X)$ induces a morphism $g_{X}: U(\operatorname{Lie}(X)) \rightarrow \operatorname{Dist}(X)$ of superalgebras.

Lemma 3.1 (see Lemma 1.2, II, §6, [6]) If char $K=0$ and $X$ is an algebraic supergroup, then $g_{X}$ is an isomorphism of Hopf superalgebras.

Proof. Let $\phi_{1}, \ldots, \phi_{n}$ form a basis of $\operatorname{Lie}(X)$ dual to a homogeneous basis $f_{1}, \ldots, f_{n}$ of the superspace $\mathcal{M} / \mathcal{M}^{2}$, where $\left|\phi_{i}\right|=\left|f_{i}\right|=0$ for $1 \leq i \leq t$ and $\left|\phi_{j}\right|=\left|f_{j}\right|$ for $t+1 \leq j \leq n$.

By induction on $l$ and using formula (3.1) of 24$]$ we infer that

$$
\begin{gathered}
\psi_{1} \ldots \psi_{l}\left(g_{1} \ldots g_{l}\right)=\sum_{1 \leq i \leq l}(-1)^{\sum_{1 \leq t<i}\left|g_{i}\right|\left|g_{t}\right|+\sum_{1<t}\left|g_{i}\right|\left|\psi_{t}\right|} \psi_{1}\left(g_{i}\right) \times \\
\sum_{\sigma(1)=i}(-1)^{\sum_{2 \leq s<t, \sigma(s)>\sigma(t)}\left|g_{\sigma(s)}\right|\left|g_{\sigma(t)}\right|+\sum_{2 \leq j<k}\left|\psi_{k}\right|\left|g_{\sigma(j)}\right|} \psi_{2}\left(g_{\sigma(2)}\right) \ldots \psi_{l}\left(g_{\sigma(l)}\right)
\end{gathered}
$$

for $\psi_{1}, \ldots, \psi_{l} \in \operatorname{Lie}(X)$ and $g_{1}, \ldots, g_{l} \in \mathcal{M}$. This implies

$$
\psi_{1} \ldots \psi_{l}\left(g_{1} \ldots g_{l}\right)=\sum_{\sigma \in S_{l}}(-1)^{\sum_{s<t, \sigma(s)>\sigma(t)}\left|g_{\sigma(s)}\right|\left|g_{\sigma(t)}\right|+\sum_{j<i}\left|\psi_{i}\right|\left|g_{\sigma(j)}\right|} \psi_{1}\left(g_{\sigma(1)}\right) \ldots \psi_{l}\left(g_{\sigma(l)}\right) .
$$

In particular,

$$
\prod_{1 \leq i \leq n} \phi_{i}^{s_{i}}\left(\prod_{1 \leq i \leq n} f_{i}^{d_{i}}\right)= \pm \delta_{s_{1}}, d_{1} \ldots \delta_{s_{n}, d_{n}} s_{1} ! \ldots s_{n} !
$$

where $l=s_{1}+\ldots+s_{n}=d_{1}+\ldots+d_{n}$ and $s_{t+1}, \ldots, s_{n}, d_{t+1}, \ldots, d_{n} \in\{0,1\}$. Comparison of dimensions shows that $g_{X}$ is an isomorphism of superalgebras. 
By definition, $\delta_{\operatorname{Dist}(X)}(\phi)\left(f_{1} \otimes f_{2}\right)=\phi\left(f_{1} f_{2}\right)=\epsilon_{X}\left(f_{1}\right) f_{2}+f_{1} \epsilon_{X}\left(f_{2}\right)$ for any $\phi \in \operatorname{Lie}(X)$, that is $\delta_{\operatorname{Dist}(X)}(\phi)=\phi \otimes 1+1 \otimes \phi$. The same formula (3.1) of [24] implies that $s_{X}(f)+f \in$ $\mathcal{M}^{2}$ for $f \in \mathcal{M}$. In particular, $s_{\text {Dist }(X)}(\phi)(f)=\phi\left(s_{X}(f)\right)=-\phi(f)$ for $\phi \in \operatorname{Lie}(X)$, that is $s_{\text {Dist }(X)}(\phi)=-\phi$. Thus $g_{X}$ is an isomorphism of filtered Hopf superalgebras.

For $A \in S A l g_{K}$ let $A\left[\varepsilon_{0}, \varepsilon_{1}\right]$ be a (commutative) superalgebra of dual numbers. By definition, $A\left[\varepsilon_{0}, \varepsilon_{1}\right]=\left\{a+\varepsilon_{0} b+\varepsilon_{1} c \mid a, b, c \in A\right\},\left|\varepsilon_{i}\right|=i, \varepsilon_{i} \varepsilon_{j}=0, i, j \in\{0,1\}$. We have two morphism of superalgebras $p_{A}: A\left[\varepsilon_{0}, \varepsilon_{1}\right] \rightarrow A$ and $i_{A}: A \rightarrow A\left[\varepsilon_{0}, \varepsilon_{1}\right]$ defined by $a+\varepsilon_{0} b+\varepsilon_{1} c \mapsto a$ and $a \mapsto a$ respectively. Define the functor $\operatorname{Lie}(G)$ as

$$
\mathbf{L i e}(G)(A)=\operatorname{ker}\left(G\left(A\left[\varepsilon_{0}, \varepsilon_{1}\right]\right) \stackrel{G\left(p_{A}\right)}{\rightarrow} G(A), A \in S A l g_{K} .\right.
$$

It is called Lie superalgebra functor of $G$. Let $V$ be a superspace. Define the functor $V_{a}$ from $S A l g_{K}$ to the category of vector superspaces, by $V_{a}(A)=V \otimes A$. The following lemma is obvious (see also [27, 29]).

Lemma 3.2 There is an isomorphism of abelian group functors $\operatorname{Lie}(G)_{a} \simeq \operatorname{Lie}(G)$ given by

$$
(v \otimes a)(f)=\epsilon_{G}(f)+(-1)^{|a||f|} \varepsilon_{|v \otimes a|} v(f) a, v \in \operatorname{Lie}(G)=\left(\mathcal{M} / \mathcal{M}^{2}\right)^{*}, a \in A, f \in K[G] .
$$

If we identify $\operatorname{Lie}(G) \otimes A$ with $\operatorname{Hom}_{K}\left(\mathcal{M} / \mathcal{M}^{2}, A\right)$ via $(v \otimes a)(f)=(-1)^{|a||f|} v(f) a$, then the above isomorphism can be represented as

$$
u \mapsto \epsilon_{G}+\varepsilon_{0} u_{0}+\varepsilon_{1} u_{1}, u \in \operatorname{Hom}_{K}\left(\mathcal{M} / \mathcal{M}^{2}, A\right) .
$$

Besides, this isomorphism induces the $A$-supermodule structure on $\operatorname{Lie}(G)(A)$. The supergroup $G$ acts on the functor $\operatorname{Lie}(G)$ by

$$
(g, x) \mapsto G\left(i_{A}\right)(g) x G\left(i_{A}\right)(g)^{-1}, g \in G(A), x \in \mathbf{L i e}(G)(A), A \in S A l g_{K} .
$$

This action is called adjoint and denoted by Ad.

Lemma 3.3 The adjoint action is linear. In particular, it induces a supergroup morphism $G \rightarrow G L(\operatorname{Lie}(G))$.

Proof. Let $u=v \otimes a \in \operatorname{Lie}(G) \otimes A$ and $g \in G(A)$. Denote the element $G\left(i_{A}\right)(g)$ by $\bar{g}$. Then

$$
\begin{gathered}
\bar{g} u \bar{g}^{-1}(f)=\sum g\left(f_{1}\right) u\left(f_{2}\right) g\left(s_{G}\left(f_{3}\right)\right)=\epsilon_{G}(f)+\sum(-1)^{|a||f|+\left|f_{1}\right||v|} \varepsilon_{|u|} g\left(f_{1}\right) v\left(f_{2}\right) g\left(s_{G}\left(f_{3}\right)\right) a= \\
\left(\bar{g}(v \otimes 1) \bar{g}^{-1} a\right)(f),
\end{gathered}
$$

where $\left(\delta_{G} \otimes 1\right) \delta_{G}(f)=\left(1 \otimes \delta_{G}\right) \delta_{G}(f)=\sum f_{1} \otimes f_{2} \otimes f_{3}, f \in K[G]$.

Lemma 3.4 The differential of Ad coincides with -ad.

Proof. Denote $\operatorname{Lie}(G)$ by $L$. We have a commutative diagram

$$
\begin{array}{ccc}
G\left(K\left[\varepsilon_{0}, \varepsilon_{1}\right]\right) & \stackrel{A d}{\rightarrow} & G L(L)\left(K\left[\varepsilon_{0}, \varepsilon_{1}\right]\right) \\
\uparrow & & \uparrow \\
\operatorname{Lie}(G) & \stackrel{d(A d)}{\rightarrow} & g l(L) .
\end{array}
$$


Notice that the image of $A \in g l(L)$ in $G L(L)\left(K\left[\varepsilon_{0}, \varepsilon_{1}\right]\right)$ is equal to $i d_{L}+\varepsilon_{0} A_{0}+\varepsilon_{1} A_{1}$. Choose $x, y \in \operatorname{Lie}(G)$. Then

$$
t=(d(\mathbf{A d})(x))(y)=\left(\epsilon_{G}+\varepsilon_{|x|} x\right)\left(\epsilon_{G}+\varepsilon_{|y|}^{\prime} y\right)\left(\epsilon_{G}-\varepsilon_{|x|} x\right),
$$

where the product is computed in $G L(L)\left(\left(K\left[\varepsilon_{0}, \varepsilon_{1}\right]\right)\left[\varepsilon_{0}^{\prime}, \varepsilon_{1}^{\prime}\right]\right)$. Further,

$$
\begin{gathered}
t(f)=\epsilon_{G}(f)+\varepsilon_{|y|}^{\prime}\left(y(f)+\varepsilon_{|x|}\left(\sum(-1)^{|x||y|} x\left(f_{1}\right) y\left(f_{2}\right) \epsilon_{G}\left(f_{3}\right)-\epsilon_{G}\left(f_{1}\right) y\left(f_{2}\right) x\left(f_{3}\right)\right)\right)= \\
\epsilon_{G}(f)+\varepsilon_{|y|}^{\prime}\left(y(f)-\varepsilon_{|x|}[y, x](f)\right) .
\end{gathered}
$$

Following [6], II, $\S 4$, we will denote the image of $u \in \operatorname{Lie}(G)(A)$ in $G\left(A\left[\varepsilon_{0}, \varepsilon_{1}\right]\right)$ by $e^{\varepsilon_{0} u_{0}+\varepsilon_{1} u_{1}}$

\section{Quotient $K$-sheafs}

Let $G$ be a group dur $K$-sheaf and assume that $G$ acts freely on a dur $K$-sheaf $X$, that is for any $R \in S A l g_{K}$ the group $G(R)$ acts freely on $X(R)$. Then the functor $R \rightarrow$ $(X / G)_{(n)}(R)=X(R) / G(R)$ satisfies the properties $\left(^{*}\right)$ and $\left(^{* *}\right)$ for ff-coverings. The proof of this fact can be copied from [4], part I (5.5). Call the above functor $(X / G)_{(n)}$ a naive quotient, the dur $K$-sheaf $(X / \tilde{\tilde{G}})_{(n)}$ a quotient dur $K$-sheaf (of $X$ by $G$ ) and denote it by $X \tilde{\tilde{/}} G$. Then $(X / G)_{(n)} \subseteq X \tilde{\tilde{/}} G$ and there is a canonical $G$-invariant morphism $\tilde{\tilde{\pi}}: X \rightarrow X \tilde{\tilde{\mu}} G$. Besides, for any other $G$-invariant morphism of dur $K$-sheafs $h: X \rightarrow Z$ there is a unique morphism $v: X \tilde{\tilde{\mu}} G \rightarrow Z$ such that $h=v \tilde{\tilde{\pi}}$ and these properties define $X \tilde{\tilde{/}} G$ uniquely up to an isomorphism. Analogous statements are valid for $K$-sheafs and there is an inclusion $X \tilde{/} G \subseteq X \tilde{\tilde{/}} G$.

Let $X$ be an affine superscheme and let $G$ be an affine supergroup acting on $X$ via $f: X \times G \rightarrow X$. If $\tau: K[X] \rightarrow K[X] \otimes K[G]$ is a comorphism dual to $f$, then $K[X]^{G}=$ $\{a \in K[X] \mid \tau(a)=a \otimes 1\}$ is a supersubalgebra of $K[X]$. The embedding $K[X]^{G} \rightarrow K[X]$ induces a $G$-invariant morphism $i: X \rightarrow S S p K[X]^{G}$. In particular, there are uniquely defined morphisms $i^{\prime}: X \tilde{/} G \rightarrow S S p K[X]^{G}$ and $i ": X \tilde{\tilde{/}} G \rightarrow S S p K[X]^{G}$ such that $i^{\prime} \tilde{\pi}=i=i " \tilde{\tilde{\pi}}$.

Proposition 4.1 Suppose that $X \tilde{\tilde{\mu}} G$ (or $X \tilde{/} G$, respectively) is an affine superscheme. Then $i "$ (or $i^{\prime}$, respectively) is an isomorphism and $K[X]$ is a faithfully flat $K[X]^{G}$ supermodule. If $X / G$ is an affine superscheme and $G$ is algebraic, then $K[X]^{G} \leq K[X]$.

Proof. Let $X \tilde{\tilde{\mu}} G=S S p R$ or $X \tilde{/} G=S S p R$. There is a canonical isomorphism $p r_{X} \times f$ : $X \times G \simeq X \times{ }_{S S p} R X$ (see [4], part I (5.5)) that is dual to the isomorphism of superalgebras $\phi: K[X] \otimes_{R} K[X] \simeq K[X] \otimes K[G]$ defined by $a \otimes b \mapsto \sum a b_{1} \otimes b_{2}$, where $\tau(b)=\sum b_{1} \otimes b_{2}$ and $a, b \in K[X]$. Repeating the proof of [4], part I (5.7) we obtain an isomorphism of $B$-superalgebras $B \otimes_{R} K[X] \simeq B \otimes K[G]$, where $B$ is either ff-covering of $R$ or $R \leq B$. Using a superversion of Proposition 4 from [3], I, $\S 3$ we see that $K[X]$ is a faithfully flat $R$-supermodule. Thus $K[X]^{G} \subseteq R \subseteq K[X]$. If $G$ is algebraic, then $B \otimes K[G]$ is a finitely 
presented $B$-superalgebra. A superversion of Lemma 1.4, [6], I implies that $R \leq K[X]$ in the case of $K$-sheafs. Composition of $\phi$ and an exact sequence

$$
0 \rightarrow R \rightarrow K[X] \rightarrow K[X] \otimes_{R} K[X]
$$

gives $R \subseteq K[X]^{G}$.

Proposition 4.2 Assume that $R$ is a supersubalgebra of $K[X]^{G}$ and the canonical morphism $X \times G \rightarrow X \times{ }_{S S p} R X$ is an isomorphism. If $K[X]$ is a faithfully flat $R$-supermodule, then $R=K[X]^{G}$ and $X \tilde{\tilde{/}} G \simeq S S p R$. Additionally, if $R \leq K[X]$, then $X \tilde{/} G \simeq S S p R$.

Proof. Consider a $G$-invariant morphism of dur $K$-sheafs $X \rightarrow Z$. By Yoneda's lemma this morphism is uniquely defined by some element $z \in Z(K[X])$. The $G$-invariance of this morphism is equivalent to the following property. For any $A \in S A \lg _{K}$ and for arbitrary $\alpha \in X(A), \beta \in G(A)$ we have $Z(\alpha)(z)=Z((\alpha \otimes \beta) \tau)(z)$. Set $A=K[X] \otimes K[G]$ and

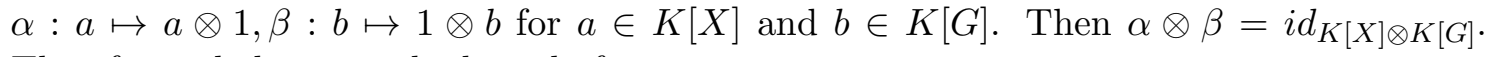
Therefore $z$ belongs to the kernel of

$$
Z(K[X]) \underset{Z(\tau)}{\stackrel{Z(\alpha)}{\rightarrow}} Z(K[X] \otimes K[G]) .
$$

On the other hand, the above diagram can be identified with

$$
Z(K[X]) \underset{Z \underset{\left(\gamma_{2}\right)}{\rightarrow}}{\stackrel{Z\left(\gamma_{1}\right)}{\rightarrow}} Z\left(K[X] \otimes_{R} K[X]\right)
$$

via bijection $Z\left(K[X] \otimes_{R} K[X]\right) \rightarrow Z(K[X] \otimes K[G])$, where $\gamma_{1}: a \mapsto a \otimes 1$ and $\gamma_{2}: a \mapsto 1 \otimes a$ for $a \in K[X]$. Setting $Z=A^{1 \mid 1}$ we obtain that $K[X]^{G} \subseteq R$. Furthermore, if $Z$ is a dur $K$ sheaf, then $z$ belongs to the image of the map $Z(R) \rightarrow Z(K[X])$ induced by the inclusion $R \rightarrow K[X]$. The above quoted Yoneda's lemma completes the proof. These arguments can be repeated per verbatim for $K$-sheafs.

\section{Coideal supersubalgebras of Hopf superalgebras}

Let $A$ be a commutative Hopf superalgebra. Its supersubalgebra $B \subseteq A$ is called a left (or right, respectively) coideal iff $B$ is a left (or right, respectively) $A$-supercomodule. A typical example is as follows. If $I$ is a superideal and a coideal of $A$, then $C=A / I$ is a superbialgebra and $B=A^{C}$ (or $B={ }^{C} A$, respectively) is a left (or right, respectively) coideal, see [12. Denote by $\mathcal{H}^{C}$ the category whose objects are right $A$-supermodules and $C$-supercomodules simultaneously, together with even morphisms such that $\tau_{M}(m a)=$ $\sum(-1)^{\left|c_{2}\right|\left|a_{1}\right|} m_{1} a_{1} \otimes c_{2} p\left(a_{2}\right)$, where $\delta_{A}(a)=\sum a_{1} \otimes a_{2}$ and $\tau_{M}(m)=\sum m_{1} \otimes c_{2}$ for $m \in M, a \in A, M \in \mathcal{H}^{C}$ and $p: A \rightarrow C=A / I$ is the canonical epimorphism. For example, $A, C \in \mathcal{H}^{C}$.

Symmetrically, let $B$ be a left coideal supersubalgebra of $A$. Denote by ${ }_{B} \mathcal{H}$ the category whose objects are left $B$-supermodules and $A$-supercomodules simultaneously, together with even morphisms such that $\tau_{M}(b m)=\sum(-1)^{\left|b_{2}\right|\left|a_{1}\right|} b_{1} a_{1} \otimes b_{2} m_{2}$, where $\delta_{A}(b)=\sum b_{1} \otimes$ $b_{2}$ and $\tau_{M}(m)=\sum a_{1} \otimes m_{2}$ for $b \in B, m \in M, M \in{ }_{B} \mathcal{H}$. For example, $B, A \in{ }_{B} \mathcal{H}$. 
Lemma 5.1 Let $N$ be a right $A$-supermodule, $B$ be a coideal superalgebra of $A$ and $M \in$ ${ }_{B} \mathcal{H}$. The linear map $\xi: N \otimes M \rightarrow N \otimes M$, defined by $\xi(n \otimes m)=\sum n a_{1} \otimes m_{2}$, induces an isomorphism of superspaces $N \otimes_{B} M \rightarrow N \otimes \bar{M}$, where $\bar{M}=M / B^{+} M$ and $B^{+}=$ $B \bigcap \operatorname{ker} \epsilon_{A}$.

Proof. It is not difficult to see that $\xi$ is an isomorphism of superspaces. The inverse $\xi^{-1}$ of $\xi$ is defined by $\xi^{-1}(n \otimes m)=\sum n s_{A}\left(a_{1}\right) \otimes m_{2}$. We have

$$
\begin{gathered}
\xi(n b \otimes m-n \otimes b m)=\sum n b a_{1} \otimes m_{2}-\sum(-1)^{\left|a_{1}\right|\left|b_{2}\right|} n b_{1} a_{1} \otimes b_{2} m_{2}= \\
-\sum(-1)^{\left|a_{1}\right|\left|b_{2}\right|} n b_{1} a_{1} \otimes\left(b_{2}-\epsilon_{A}\left(b_{2}\right)\right) m_{2} \in N \otimes B^{+} M
\end{gathered}
$$

and

$\xi^{-1}(n \otimes b m)=\sum(-1)^{\left|a_{1}\right|\left|b_{2}\right|} n s_{A}\left(b_{1} a_{1}\right) \otimes b_{2} m_{2}=-\left(\sum n s\left(a_{1} b_{1}\right) b_{2} \otimes m_{2}-\sum n s\left(a_{1} b_{1}\right) \otimes b_{2} m_{2}\right)$.

Lemma 5.2 Let $p: A \rightarrow C$ be an epimorphism of superbialgebras. For any $N \in \mathcal{H}^{C}, M \in$ $A$-scomod the previously defined map $\xi$ induces an isomorphism of superspaces $N^{C} \otimes M \simeq$ $N \square_{C} M$.

Proof. It can be checked easily that $\xi\left(N^{C} \otimes M\right) \subseteq N \square_{C} M$ and therefore it remains to show that $\xi^{-1}\left(N \square_{C} M\right) \subseteq N^{C} \otimes M$. Let $\left\{m_{i}\right\}_{i \in I}$ be a homogeneous basis of $M$ and $\tau_{M}\left(m_{i}\right)=\sum_{k \in I} a_{i k} \otimes m_{k}$ for $i \in I$. Notice that $\left|a_{i j}\right|=\left|m_{i}\right|+\left|m_{j}\right|(\bmod 2)$ for $i, j \in I$. The condition $\sum_{i \in I} n_{i} \otimes m_{i} \in N \square_{C} M$ is equivalent to equalities $\tau_{N}\left(n_{i}\right)=\sum_{k \in I} n_{k} \otimes p\left(a_{k i}\right)$ for $i \in I$. Applying $\xi^{-1}$ we see that all we have to check is $u_{k}=\sum_{i \in I} n_{i} s_{A}\left(a_{i k}\right) \in N^{C}$ for all $k$. But this follows from

$$
\begin{gathered}
\tau_{N}\left(u_{k}\right)=\sum_{i, t, l \in I}(-1)^{\left|a_{l t}\right|\left|a_{l k}\right|} n_{t} s_{A}\left(a_{l k}\right) \otimes p\left(a_{t i} s_{A}\left(a_{i l}\right)\right)= \\
\sum_{t, l \in I}(-1)^{\left|a_{l t}\right|\left|a_{l k}\right|} n_{t} s_{A}\left(a_{l k}\right) \otimes p\left(\epsilon\left(a_{t l}\right)\right)=u_{k} \otimes p(1) .
\end{gathered}
$$

Observe that a superideal $A B^{+}$is also a coideal. It follows from $\delta_{A}(b)=b \otimes 1+\sum a_{1} \otimes b_{2}$ for $b \in B, b_{2} \in B^{+}$. Define functors $\Phi:{ }_{B} \mathcal{H} \rightarrow \bar{A}-\operatorname{scomod}$ and $\Psi: \bar{A}-\operatorname{scomod} \rightarrow{ }_{B} \mathcal{H}$ by $\Phi(M)=\bar{M}, \Psi(N)=A \square_{\bar{A}} N$. The functor $\Psi$ is right adjoint of $\Phi$ by [12]. Adjunctions $u_{M}: M \rightarrow \Psi \Phi(M)=A \square_{\bar{A}} \bar{M}, v_{N}: \Phi \Psi(N)=\overline{A \square_{\bar{A}} N} \rightarrow N$ are defined by $m \mapsto \sum a_{1} \otimes \overline{m_{2}}$ and $\overline{\sum n \otimes a} \mapsto \sum \epsilon_{A}(a) n$. Symmetrically, one can define functors $\Theta: \operatorname{smod}-B \rightarrow \mathcal{H}^{C}$ and $\Omega: \mathcal{H}^{C} \rightarrow$ smod $-B$ by $\Theta(M)=M \otimes_{B} A, \Omega(N)=N^{C}$. The functor $\Theta$ is left adjoint of $\Omega$ by [12]. The corresponding adjunctions $f_{M}: M \rightarrow \Omega \Theta(M)=\left(M \otimes_{B} A\right)^{C}$ and $g_{N}: \Theta \Omega(N)=N^{C} \otimes_{B} A \rightarrow N$ are defined by $f_{M}(m)=m \otimes 1$ and $g_{N}(n \otimes a)=n a$.

Lemma 5.3 Let $V \in \operatorname{smod}-A$ be a flat $B$-supermodule. Then $V \otimes_{B} \Psi(N)$ is isomorphic to $V \otimes N$ via $v \otimes\left(\sum a \otimes n\right) \mapsto \sum v a \otimes n$, where $v \in V, \sum a \otimes n \in \Psi(N)$.

Proof. Maps $\left(i d_{A} \otimes ?\right) \delta_{A} \otimes i d_{N}$ and $i d_{A} \otimes \tau_{N}$ are morphisms of left $B$-supermodules. They combine to a canonical isomorphism $V \otimes_{B} \Psi(N) \simeq\left(V \otimes_{B} A\right) \square_{\bar{A}} N$ (see also Proposition 1.3, [13]). On the other hand, $\xi: V \otimes_{B} A \rightarrow V \otimes \bar{A}$ is an isomorphism of right $\bar{A}$-supercomodules and its composition with $\xi \otimes i d_{N}$ gives $V \otimes_{B} \Psi(N) \simeq V \otimes \bar{A} \square_{\bar{A}} N \simeq V \otimes N$. 
Lemma 5.4 Let $V \in A-$ scomod and $V$ be a coflat $C$-supercomodule. For any $B$ supermodule $T$ there is an isomorphism $T \otimes V \simeq \Theta(T) \square_{C} V$ defined by $t \otimes v \mapsto \sum t \otimes c_{1} \otimes v_{2}$, where $\tau_{V}(v)=\sum c_{1} \otimes v_{2}$.

Proof. Denote by $p: T \otimes A \rightarrow T \otimes_{B} A$ the canonical epimorphism of superspaces. A sequence $0 \rightarrow \operatorname{ker} p \rightarrow T \otimes A \stackrel{p}{\rightarrow} T \otimes_{B} A$ is exact in the category $C-$ scomod. In particular, it induces a canonical isomorphism $T \otimes_{B}\left(A \square_{C} V\right) \rightarrow\left(T \otimes_{B} A\right) \square_{C} V$. Composition with $i d_{T} \otimes \xi$ gives the isomorphism of the lemma.

Lemma 5.5 Let $A$ be a superalgebra and let $\phi: M \rightarrow N$ be an epimorphism of free $A$-supermodules of the same finite rank. Then $\phi$ is an isomorphism.

Proof. Denote the rank of both $M$ and $N$ by $(r, s)$. Choose free generators $m_{i}, n_{i}$ for $1 \leq i \leq r+s=n$ of supermodules $M$ and $N$ correspondingly. Additionally, assume that $\left|m_{i}\right|=\left|n_{i}\right|=0$ if $1 \leq i \leq r$ and $\left|m_{i}\right|=\left|n_{i}\right|=1$ otherwise. Denote $\phi\left(m_{i}\right)=n_{i}^{\prime}$ for $1 \leq i \leq n$ and write $n_{i}^{\prime}=\sum_{1 \leq k \leq n} b_{i k} n_{k}, n_{i}=\sum_{1 \leq k \leq n} c_{i k} n_{k}^{\prime}$ for $1 \leq i \leq n$. Matrices $B=\left(b_{i k}\right)$ and $C=\left(c_{i k}\right)$ belong to $M a t_{r, s}(A)_{0}$ and $C B=E_{n}$. Considering the last matrix equation modulo the superideal $A A_{1}$, we infer that even diagonal blocks of the matrix $C$ are invertible. By Lemma 1.7.2 of [8], (see also Theorem 3.1, [16]) $C$ is invertible. In particular, the elements $n_{i}^{\prime}$ are free generators over $A$.

Proposition 5.1 If $M \in \mathcal{H}_{B}$, then a left $B$-module $M_{B^{+}}$is flat.

Proof. We follow the same ideas as in the proofs of Lemma 3.1 and of Corollary 3.3 in [10]. First of all, it is enough to consider the case when $M$ is finitely generated. Using the isomorphism $K[G] \otimes_{B} M \rightarrow K[G] \otimes \bar{M}$ we see that a left $K[G]$-supermodule $K[G] \otimes_{B} M$ is free of $\operatorname{rank}(m, n)$, where $m=\operatorname{dim} \bar{M}_{0}$ and $n=\operatorname{dim} \bar{M}_{1}$. It is clear that there is an isomorphism of $B$-supermodules $f: B^{m} \bigoplus\left(B^{c}\right)^{n} \rightarrow M$ which induces an isomorphism modulo $B^{+}$. On the other hand, $B_{B^{+}}$is a local algebra. Thus $\operatorname{rad}_{B^{+}}=$ $\left(B^{+} B_{B^{+}}\right) M_{B^{+}}$and the localization of $f$, denoted by $f_{B^{+}}: B_{B^{+}}^{m} \bigoplus\left(B_{B^{+}}^{c}\right)^{n} \rightarrow M_{B^{+}}$, is an epimorphism (cf. [15, 9.3.5, 9.4.1). Tensoring with $K[G]_{B^{+}}$produces an epimorphism $g: K[G]_{B^{+}}^{m} \bigoplus\left(K[G]_{B^{+}}^{c}\right)^{n} \rightarrow K[G]_{B^{+}} \otimes_{B_{B^{+}}} M_{B^{+}}$. The $K[G]_{B^{+-}}$-supermodule $K[G]_{B^{+}} \otimes_{B_{B^{+}}}$ $M_{B^{+}}$can be naturally identified with $K[G]_{B^{+}} \otimes_{B} M \simeq K[G]_{B^{+}} \otimes \bar{M}$. By Lemma 5.5, the map $g$ is an isomorphism and its composition with $B_{B^{+}}^{m} \bigoplus\left(B_{B^{+}}^{c}\right)^{n} \rightarrow\left(K[G]_{B^{+}}^{c}\right)^{n} \rightarrow$ $K[G]_{B^{+}}$is identified with $f_{B^{+}}$.

Lemma 5.6 A Hopf superalgebra $H$ is a direct union of all of its finitely generated Hopf supersubalgebras.

Proof. Any finite subset $X \subseteq H$ is contained in a finite dimensional supersubcoalgebra $C$. Let $c_{i}$ for $1 \leq i \leq t$ be a homogeneous basis of $C$. The supersubalgebra generated by $c_{i}$ and $s_{H}\left(c_{i}\right)$ for $1 \leq i \leq t$ is obviously a Hopf superalgebra containing $X$.

Theorem 5.1 The Hopf superalgebra $A$ is a flat left B-module.

Proof. Without a loss of generality one can assume that $K$ is algebraically closed and by Lemma 5.6 one can suppose that $A$ is finitely generated. For any $\mathcal{M} \in \operatorname{Max}(A)$, the map $T_{\mathcal{M}}: A \rightarrow A$ defined by $T_{\mathcal{M}}(f)=\sum \pi_{\mathcal{M}}\left(f_{1}\right) f_{2}$, where $\delta_{A}(f)=\sum f_{1} \otimes f_{2}$ and $\pi_{\mathcal{M}}: A \rightarrow A / \mathcal{M}=K$, is an automorphism of the superalgebra $A$ because $A_{1} \subseteq \mathcal{M}$. The inverse of $T_{\mathcal{M}}$ is $T_{s_{A}(\mathcal{M})}$ because $\pi_{s_{A}(\mathcal{M})}(f)=\pi_{\mathcal{M}}\left(s_{A}(f)\right)$ for $f \in A$. 
Next, the map $T_{\mathcal{M}}$ takes $B$ to $B$ and $\mathcal{M}$ to $\mathcal{M}_{1}=\operatorname{ker} \epsilon_{A}$. Using Proposition 5.1, Lemma 1.2 and the left-hand side version of Proposition 8, I, $\S 2$ of [3] we see that $A_{B^{+}} \otimes_{A} A_{\mathcal{M}_{1}}$ is a flat left $B$-module. The canonical epimorphism of left $B$-modules $A_{B^{+}} \otimes_{A} A_{\mathcal{M}_{1}} \rightarrow A_{\mathcal{M}_{1}}$ is split. Therefore $A_{\mathcal{M}_{1}}$ is a flat left $B$-module. The isomorphism $T_{\mathcal{M}}$ takes this module to $A_{\mathcal{M}}^{\alpha_{\mathcal{M}}}$, where $\alpha_{\mathcal{M}}=\left.T_{\mathcal{M}}\right|_{B}$. We complete the proof by combining Proposition 1.1, the remark after Corollary 1.2 and the same reductions as in [10] (see also Lemma 7.1, III, $\S 3$ of $[6])$.

Corollary 5.1 If $B$ is a Hopf supersubalgebra of $A$, then $A$ is a faithfully flat (left and right) $B$-module.

Proof. By Theorem 5.1 it remains to show that $\mathcal{M} A \neq A$ for all $\mathcal{M} \in \operatorname{Max}(B)$. Since $J=A A_{1}$ is a Hopf superideal and $J \cap B \subseteq \mathcal{M}$, it suffices to check that $\mathcal{M}(A / J) \neq A / J$. On the other hand, $A / J$ is a Hopf algebra and $B / J$ is its Hopf subalgebra. By Takeuchi's theorem (see [12]), $A / J$ is a faithfully flat $B / J$-module. The right-hand side statement can be deduced from the right-hand side version of Theorem 5.1 .

Let $G$ be an affine supergroup and let $H$ be its closed supersubgroup. The supergroup $H$ is called faithfully exact in $G$ if $K[G]$ is a faithfully exact right $K[H]$-supercomodule. We identify the category of right $H$-supermodules $\bmod -H$ with $K[H]-$ scomod. In particular, the functor $\Psi=K[G] \square_{K[H]}$ ? can be identified with the inducing functor $i n d_{H}^{G}$ (see [1]).

Proposition 5.2 The quotient $G \tilde{\tilde{F}} H$ is affine iff $I_{H}=K[G] R^{+}$and $K[G]$ is a faithfully flat $R$-supermodule, where $R$ is a left coideal supersubalgebra of $K[G]^{H}$. If $G$ is algebraic, then $G \tilde{/ H}$ is affine iff $I_{H}=K[G] R^{+}$and $R \leq K[G]$.

Proof. Lemma 5.1 and the equality $I_{H}=K[G] R^{+}$guarantees that $G \times H \simeq G \times{ }_{S S p R} G$. Conversely, assume that the quotient $G \tilde{\tilde{/}} H$ (or $G \tilde{/} H$ ) is affine. In the following commutative diagram of superalgebras

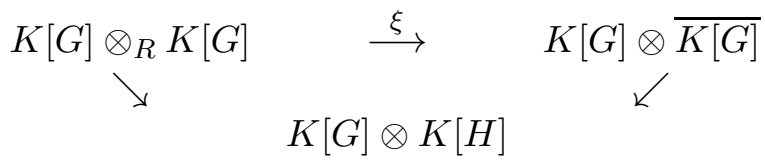

the horizontal and the left diagonal arrows are isomorphisms. Thus $\overline{K[G]} \rightarrow K[H]$ is an isomorphism and it remains to refer to Proposition 4.2.

Theorem 5.2 The following statements are equivalent :

i) Quotient $G \tilde{\tilde{/}} H$ is affine;

ii) $H$ is a faithfully exact supersubgroup of $G$;

iii) ind $_{H}^{G}$ induces an equivalence of $\bmod -H$ with the full subcategory ${ }_{R} \mathcal{H}$;

iv) $K[G]$ is an injective cogenerator in the category $\bmod -H$.

Proof. Combining Lemmas 5.1 - 5.4 and Proposition 5.2 with the proof of Theorems 1 and 2 from [12] we easily obtain that the properties i), ii) and iii) are equivalent to each other. The equivalence of iii) and iv) was proved in Lemma 1.7. 


\section{Quotients by normal supersubgroups}

A group $K$-subfunctor $H$ of a group $K$-functor $G$ is said normal if $H(A) \unlhd G(A)$ for all $A \in S A l g_{K}$. If $G$ is a dur $K$-sheaf (or $K$-sheaf, respectively) and $H$ is its normal subsheaf, then Lemma 2.3 implies that $G \tilde{\tilde{F}} H$ (or $G \tilde{/} H$, respectively) is again group dur $K$-sheaf (or group $K$-sheaf, respectively) and the canonical morphism $G \rightarrow G \tilde{\tilde{I}} H$ (or $G \rightarrow G \tilde{/} H$, respectively) is a morphism of group $K$-functors.

If $G$ is an affine supergroup and $H$ is its closed supersubgroup, then $H \unlhd G$ iff it satisfies one of the following conditions

$$
\nu_{r}(f)=\sum(-1)^{\left|f_{1}\right|\left|f_{2}\right|} f_{2} \otimes f_{1} s_{G}\left(f_{3}\right) \in I_{H} \otimes K[G],
$$

or

$$
\nu_{l}(f)=\sum(-1)^{\left|f_{1}\right|\left|f_{2}\right|} f_{2} \otimes s_{G}\left(f_{1}\right) f_{3} \in I_{H} \otimes K[G],
$$

for any $f \in I_{H}$. The first condition is called right normality condition and the second one is called left normality condition. These conditions are different in general (say for quantum groups) but for supergroups they are equivalent because $s_{G}$ is an automorphism of superalgebra $K[G]$ of order two (see [9], 1.5, and [1], §2). The morphism $\nu_{l}$ is a superalgebra morphism, it is dual to the morphism of superschemes $G \times G \rightarrow G$ given by $\left(g_{1}, g_{2}\right) \mapsto g_{2}^{-1} g_{1} g_{2}$ for $g_{1}, g_{2} \in G(A)$ and $A \in S A l g_{K}$ (which defines a right action of $G$ on itself by conjugations). Symmetrically, $\nu_{r}$ is dual to the morphism $\left(g_{1}, g_{2}\right) \mapsto g_{2} g_{1} g_{2}^{-1}$. From now on, all group $K$-functors are assumed affine and all group subfunctors are assumed closed unless otherwise stated.

Lemma 6.1 If $H \unlhd G$, then Lie $(H)$ is a Lie superideal of Lie $(G)$.

Proof. It is clear that $\operatorname{Ad}(G) \subseteq \operatorname{Stab}_{G L(\operatorname{Lie}(G))}(\operatorname{Lie}(H))$. Lemma 3.4 concludes the proof.

Let $H$ be a supersubgroup of $G$. Denote the normalizer of $H$ in $G$ by $N_{G}(H)$. By definition,

$N_{G}(H)(A)=\left\{g \in G(A) \mid\right.$ for any $A$ - superalgebra $\left.B G\left(\iota_{A}^{B}\right)(g) H(B) G\left(\iota_{A}^{B}\right)(g)^{-1}=H(B)\right\}$.

Proposition 6.1 The normalizer $N_{G}(H)$ is a closed supersubgroup.

Proof. Consider $f \in I_{H}$. Let $\nu_{l}(f)=\sum u_{1} \otimes u_{2}$ modulo $I_{H} \otimes K[G]$, where $u_{1}$ are linearly independent modulo $I_{H}$. The elements $u_{2}$ are called companions of $f$. Let $I$ be a superideal of $K[G]$ generated by companions of all $f$. Set $B=K[H] \otimes A$ and $\iota_{A}^{B}=1 \otimes i d_{A}$. Then $h=p \otimes 1 \in H(B)$, where $p$ is the canonical epimorphism $K[G] \rightarrow K[H]$. We have

$$
\left(G\left(\iota_{A}^{B}\right)(g)^{-1} h G\left(\iota_{A}^{B}\right)(g)\right)(f)=\sum u_{1} \otimes g\left(u_{2}\right)=0 .
$$

Thus $g\left(u_{2}\right)=0$. Conversely, if all $g\left(u_{2}\right)=0$, then $G\left(\iota_{A}^{B}\right)(g) H(B) G\left(\iota_{A}^{B}\right)(g)^{-1} \subseteq H(B)$ for any $A$-superalgebra $B$. It implies $N_{G}(H)=V\left(I+s_{G}(I)\right)$.

Lemma 6.2 If $H \unlhd G$, then $R={ }^{H} K[G]=K[G]^{H}$ is a Hopf supersubalgebra of $K[G]$. 
Proof. It is enough to observe that $f \in R=K[G]^{H}$ iff $f(g h)=f(g)$ for all $g \in G(A), h \in$ $H(A)$ and $A \in S A l g_{K}$. In particular, $f(h g)=f\left(g g^{-1} h g\right)=f(g)$ implies that the antipode $s_{G}$ induces an automorphism of $R$.

If $\phi: G \rightarrow H$ and $\psi: L \rightarrow H$ are morphisms of affine supergroups, then $G \times_{H} L$ is a supersubgroup of $G \times L$. In the case $L \leq H$ we can identify the fiber product $G \times{ }_{H} L$ with a supersubgroup of $G$ that we call a preimage of $\phi^{-1}(L)$. Besides, $K\left[\phi^{-1}(L)\right]=$ $K[G] / K[G] \phi^{*}\left(I_{L}\right)$. In particular, a kernel $N=\operatorname{ker} \phi \leq G$ coincides with $G \times_{H} E$, where $E$ is the trivial supersubgroup of $H$. It is also a kernel in the category of supergroups (see Proposition 1.6.1 from [9]). As in [9], we have that $N \unlhd G$ and, moreover, $N \unlhd \phi^{-1}(L)$.

Theorem 6.1 A supergroup $G \tilde{\tilde{F}} N$ is isomorphic to a supersubgroup of $H$ (the image of $\phi)$. More precisely, $\operatorname{Im} \phi=S S p K[H] / \operatorname{ker} \phi^{*}=S S p \operatorname{Im} \phi^{*}$. Thus $N$ is faithfully exact supersubgroup of $G$. If $G$ and $H$ are algebraic, then $G \tilde{/} N=G \tilde{\tilde{N}} N$.

Proof. The definition of $I_{N}$ guarantees that the canonical morphism $G \times N \rightarrow G \times{ }_{\operatorname{Im} \phi} G$ is an isomorphism. Besides, $\operatorname{Im} \phi^{*} \subseteq K[G]^{N}$. Proposition 5.2 and Corollary 5.1 imply the first statement. The second statement is deduced easily from Lemma 1.1.

Corollary 6.1 The canonical epimorphism $\phi^{-1}(L) \rightarrow L \bigcap \operatorname{Im} \phi$, which is dual to the embedding $K[H] /\left(I_{L}+\operatorname{ker} \phi^{*}\right) \rightarrow K[G] / K[G] \phi^{*}\left(I_{L}\right)$, induces an isomorphism $\phi^{-1}(\tilde{\tilde{L}}) / N \simeq$ $L \bigcap \operatorname{Im} \phi$. Moreover, the diagram

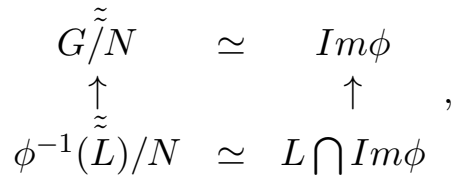

where the vertical maps are natural embeddings, is commutative.

Proposition 6.2 The following statements are equivalent:

i) A quotient $G \tilde{\tilde{/}} H$ is affine for any algebraic supergroup $G$ and for any normal supersubgroup $H$ of $G$;

ii) Quotient $G \tilde{\tilde{/}} H$ is affine for any affine supergroup $G$ and for any normal supersubgroup $H$ of $G$.

Proof. We have to check only the implication i) $\rightarrow$ ii). According to Lemma 5.6, $K[G]$ is a direct union of its finitely generated Hopf supersubalgebras, say $K[G]=\bigcup_{\alpha \in \mathcal{A}} B_{\alpha}$. Set $I_{\alpha}=B_{\alpha} \bigcap I_{H}$ for $\alpha \in \mathcal{A}$. By Theorem 5.1 and by i) for any pair $H_{\alpha}=S S p B_{\alpha} / I_{\alpha} \unlhd G_{\alpha}=$ $S S p B_{\alpha}$ we obtain that $B_{\alpha}=K\left[G_{\alpha}\right]$ is a faithfully flat (left and right) $R_{\alpha}=K\left[G_{\alpha}\right]^{H_{\alpha_{-}}}$ module and $I_{\alpha}=B_{\alpha} R_{\alpha}^{+}$. It is clear that $\bigcup_{\alpha \in \mathcal{A}} R_{\alpha}=R=K[G]^{H}$. By Lemma 7.1, III, $\S 3$ of $[6], K[G]$ is faithfully flat (left and right) $R$-module. It remains to observe that $I_{H}=\bigcup_{\alpha \in \mathcal{A}} I_{\alpha}=\bigcup_{\alpha \in \mathcal{A}} B_{\alpha} R_{\alpha}^{+}=K[G] R^{+}$.

Remark 6.1 If $G$ is algebraic, $H \unlhd G$ and $G \tilde{\tilde{F}} H$ is affine, then $G \tilde{\tilde{/}} H=G \tilde{/} H$. In fact, $I_{H}=K[G] R^{+}$, where $R=K[G]^{H}$. By Lemma 1.1 the superideal $I_{H}$ is finitely generated. Moreover, it is generated by some finite subset from $R^{+}$. By Lemma 5.6 this subset is contained in a finitely generated Hopf supersubalgebra $B \subseteq R$. Using Propositions 5.2 and 4.2 we see that $B=R$. 
Proposition 6.3 Let $G$ be an algebraic supergroup and $H \leq G$. There is a linear representation $\phi: G \rightarrow G L(V)$ such that $\phi: G \simeq \operatorname{Im} \phi$ and $\left.\phi\right|_{H}: H \simeq \operatorname{Stab}_{G}(W)$ for a suitable supersubspace $W \subseteq V$.

Proof. There is a finite dimensional supersubcomodule $V \subseteq K[G]$ containing all generators of $K[G]$ as well as all generators of $I_{H}$. Let $v_{1}, \ldots, v_{m+n}$ be a basis of $V$ such that $\left|v_{i}\right|=0$ for $1 \leq i \leq m$ and $\left|v_{i}\right|=1$ otherwise. Additionally, assume that $v_{1}, \ldots, v_{s}, v_{m+1}, \ldots, v_{m+t}$ for $s \leq m$ and $t \leq n$ is a basis of $W=I_{H} \cap V$. We have a morphism of supergroup $\phi: G \rightarrow G L(V)$ defined by $g \mapsto\left(g\left(r_{i j}\right)\right)$ for $g \in G(A)$ and $A \in S A l g_{K}$, where $\tau_{V}\left(v_{i}\right)=$ $\delta_{G}\left(v_{i}\right)=\sum_{1 \leq j \leq m+n} v_{j} \otimes r_{j i}$ for $1 \leq i \leq m+n$. If $M=\{1, \ldots, s, m+1, \ldots m+t\}$ and $i \in M, j \notin M$, then $r_{j i} \in I_{H}$. Superalgebra $\operatorname{Im} \phi^{*}$ is generated by the elements $r_{i j}$ and by the multiplicative set generated by determinants of even blocks of the matrix $\left(r_{i j}\right)$. On the other hand, $v_{i}=\sum_{1 \leq j \leq m+n} \epsilon_{G}\left(v_{j}\right) r_{j i}$ for every $i$. Thus $\operatorname{Im} \phi^{*}=K[G]$ and $\operatorname{ker} \phi=E$. Finally, if $i \in M$, then $v_{i}=\sum_{j \notin M} \epsilon_{G}\left(v_{j}\right) r_{j i}$. In other words, $\phi(H)=\operatorname{Stab}_{G}(W)$.

Proposition 6.4 Let $G$ be a group $K$-sheaf and $N_{1} \leq N_{2}$ be group subsheafs of $G$. If $N_{1} \unlhd$ $G$, then $H=N_{2} \tilde{/} N_{1}$ is a group subsheaf of $M=G \tilde{/ N} N_{1}$ and $M \tilde{/} H \simeq G \tilde{/} N_{2}$. Additionally, if $N_{2} \unlhd G$, then $H \unlhd M$ and the last isomorphism is an isomorphism of group sheafs. Analogous statements are valid for dur $K$-sheafs.

Proof. It is an easy consequence of the universal property of quotients combined with Lemma 2.3.

Now we can formulate and prove the main result of this paper.

Theorem 6.2 If $G$ is an affine supergroup and $N$ is a normal supersubgroup of $G$, then $G \tilde{\tilde{}} N$ is again an affine supergroup.

Let $G$ be an affine supergroup and $N$ is a normal supersubgroup of $G$. By Proposition 6.2 one can assume that $G$ is algebraic. Define the supersubgroup $\bar{N} \leq G$ in such way that $I_{\bar{N}}=K[G] R^{+}$, where $R=K[G]^{N}$. By Lemma 6.2 we have $N \leq \bar{N} \unlhd G$ and $G \tilde{\tilde{/ N}} \simeq S S p R$. It remains to prove that $N=\bar{N}$.

Lemma 6.3 The superalgebra $K[\bar{N}]^{N}$ coincides with $K$.

Proof. The canonical isomorphism $K[G] \otimes_{R} K[G] \rightarrow K[G] \otimes K[\bar{N}]$ is an isomorphism of right $K[N]$-supercomodules. Consider the following exact sequence of $R$-supermodules

$$
0 \rightarrow R \rightarrow K[G] \stackrel{\phi}{\rightarrow} K[G] \otimes K[N],
$$

where $\phi(a)=\sum a_{1} \otimes \overline{a_{2}}-a \otimes \overline{1}$ for $a \in K[G]$ and $\delta_{G}(a)=\sum a_{1} \otimes a_{2}$. Using Theorem 5.1 we obtain $K[G] \otimes_{R} R=\left(K[G] \otimes_{R} K[G]\right)^{N} \simeq K[G] \otimes K[\bar{N}]^{N}$ which implies $K[\bar{N}]^{N}=K$.

From now on we assume that $G$ is algebraic and $K[G]^{N}=K$ unless stated otherwise. Without a loss of generality one can assume that $K$ is algebraically closed. Up to the end of this section $\operatorname{char} K=p>0$.

The radical $r$ of the superalgebra $K[G]$ is a Hopf superideal. In fact, a superalgebra $K[G] / r \otimes K[G] / r$ is reduced as the coordinate algebra of an affine variety $\operatorname{Max}(K[G]) \times$ $\operatorname{Max}(K[G])$. A supergroup $G_{r e d}$ corresponding to the Hopf superideal $r$ is pure even. In other words, it is an affine group (= affine group scheme). Besides, $G_{r e d} \leq G_{e v}$, where $I_{G_{e v}}=K[G] K[G]_{1}$. It is clear that $U=N \cap G_{r e d} \unlhd G_{r e d}$. 
Lemma 6.4 We have $K\left[G_{r e d}\right]^{N_{r e d}}=K$. In particular, $G_{r e d} \leq U$ or equivalently, $U=$ $N_{\text {red }}$.

Proof. Assume that an element $f \in K[G]_{0}$ represents a $N_{\text {red }}$-invariant in $K\left[G_{r e d}\right]$, that is $\delta_{G}(f)-f \otimes 1 \in r \otimes K[G]+K[G] \otimes\left(r+I_{N}\right)$. Since $r$ is a nilpotent ideal, it follows that for a sufficiently large integer $M>0$ we have $\delta\left(f^{p^{M}}\right)-f^{p^{M}} \otimes 1 \in K[G] \otimes I_{N}$. In particular, $f^{p^{M}}=a \in K$. Thus $f=b+x$, where $b \in K, b^{p^{M}}=a$ and $x \in r$. The second statement follows from Theorem 4.3 of [11].

According to Proposition 6.3 we can write $G \leq G L(V)$ and $N=S t a b_{G}(W)$ for suitable supersubspaces $W \subseteq V$. Using the notations from Proposition 6.3 one can depict a matrix from $G L(V)(F)$ for $F \in S A l g_{K}$ as

$$
\left(\begin{array}{cc}
A & B \\
C & D
\end{array}\right)
$$

where $A=\left(A_{i j}\right) \in G L_{m}\left(F_{0}\right), B=\left(B_{i j}\right) \in M_{m \times n}\left(F_{1}\right), C=\left(C_{i j}\right) \in M_{n \times m}\left(F_{1}\right)$ and $D=$ $\left(D_{i j}\right) \in G L_{n}\left(F_{0}\right)$ for $i, j=1,2$, where the blocks $A_{i j}$ and $B_{i j}\left(C_{i j}\right.$ and $D_{i j}$, respectively) have $s$ rows if $i=1$, and $m-s$ rows if $i=2(t$ rows if $i=1$, and $n-t$ rows if $i=2$, respectively). Symmetrically, the blocks $A_{i j}$ and $C_{i j}\left(B_{i j}\right.$ and $D_{i j}$, respectively) have $s$ columns if $j=1$, and $m-s$ columns if $j=2(t$ columns if $j=1$, and $n-t$ columns if $j=2$, respectively). The supergroup $S(F)=\left(\operatorname{Stab}_{G L(V)}(W)\right)(F)$ consists of all matrices with $A_{21}=0, B_{21}=0, C_{21}=0$ and $D_{21}=0$.

The open subfunctor $G L(V)_{f}$, where $f=\operatorname{det}\left(A_{11}\right) \operatorname{det}\left(D_{11}\right)$, contains $S$. Let $U$ be a closed supersubscheme of $G L(V)_{f}$ defined by equations $A_{11}=E_{s}, A_{22}=E_{m-s}, D_{11}=$ $E_{t}, D_{22}=E_{n-t}, B_{11}=0, B_{12}=0, B_{22}=0, C_{11}=0, C_{12}=0, C_{22}=0, D_{12}=0$ and $A_{12}=0$. It is obvious that $U$ is an (unipotent) supersubgroup of $G L(V)$.

Proposition 6.5 We have an isomorphism of superschemes $\psi: G L(V)_{f} \simeq U \times S$ commuting with the right action of $S$ given by multiplication.

Proof. The above isomorphism $\psi: G L(V)_{f} \rightarrow U \times S$ is defined by the rule

$$
\left(\begin{array}{cc}
A & B \\
C & D
\end{array}\right) \mapsto\left(\begin{array}{cc}
A^{\prime} & B^{\prime} \\
C^{\prime} & D^{\prime}
\end{array}\right) \times\left(\begin{array}{cc}
A^{\prime \prime} & B " \\
C " & D^{\prime \prime}
\end{array}\right)
$$

where

$$
\left(\begin{array}{cc}
A^{\prime} & B^{\prime} \\
C^{\prime} & D^{\prime}
\end{array}\right) \in U,\left(\begin{array}{cc}
A^{\prime \prime} & B^{\prime \prime} \\
C^{\prime \prime} & D^{\prime \prime}
\end{array}\right) \in S
$$

and

$$
\begin{aligned}
& A_{11}=A{ }_{11}, A_{12}=A{ }_{12}, B_{11}=B{ }_{11}, B_{12}=B{ }_{12} \text {, } \\
& C_{11}=C{ }_{11}, C_{12}=C{ }_{12}, D_{11}=D^{\prime}{ }_{11}, D_{12}=D^{\prime}{ }_{12} \text {, } \\
& \left(\begin{array}{ll}
A_{21}^{\prime} & B_{21}^{\prime}
\end{array}\right)=\left(\begin{array}{ll}
A_{21} & B_{21}
\end{array}\right)\left(\begin{array}{ll}
A_{11} & B_{11} \\
C_{11} & D_{11}
\end{array}\right)^{-1} \\
& \left(\begin{array}{ll}
C_{21}^{\prime} & D_{21}^{\prime}
\end{array}\right)=\left(\begin{array}{ll}
C_{21} & D_{21}
\end{array}\right)\left(\begin{array}{ll}
A_{11} & B_{11} \\
C_{11} & D_{11}
\end{array}\right)^{-1}, \\
& A^{\prime \prime}{ }_{22}=A_{22}-A_{21}^{\prime} A_{12}-B_{21}^{\prime} C_{12}, B^{\prime \prime}{ }_{22}=B_{22}-A_{21}^{\prime} B_{12}-B_{21}^{\prime} D_{12} \text {, } \\
& C^{\prime \prime}{ }_{22}=C_{22}-C_{21}^{\prime} A_{12}-D_{12}^{\prime} C_{12}, D^{\prime \prime}{ }_{22}=D_{22}-C_{21}^{\prime} B_{12}-D_{12}^{\prime} D_{12} \text {. }
\end{aligned}
$$

The inverse morphism is just the multiplication map. 
Lemma 6.5 The supergroup $G$ is a closed supersubscheme of $G L(V)_{f}$.

Proof. Since $N \subseteq G L(V)_{f}$, we see that the image of $f$ in $K[G]$ is invertible modulo the superideal $I_{N}$. On the other hand, $I_{N} \subseteq r$ and therefore, $f \in K[G]^{*}$.

Proposition 6.6 The supergroup $G$ coincides with $N$.

Proof. The naive quotient morphism $G L(V)_{f} \rightarrow G L(V)_{f} / S$ can be identified with the composition of $\psi$ and the projection $U \times S \rightarrow U$. In particular, $G L(\tilde{\tilde{V}})_{f} / S=G L(V)_{f} / S \simeq$ $U$. The induced morphism $\pi: G \rightarrow G L(V)_{f} / S$ is dual to the composition of the embedding $K[U] \otimes 1 \rightarrow K[U] \otimes K[S]$ and the epimorphism $K[U] \otimes K[S] \rightarrow K[G]$. The last epimorphism is a morphism of right $K[N]$-supercomodules. Since $K[U] \otimes 1=(K[U] \otimes K[S])^{S}$ it follows that $\operatorname{Im} \pi^{*}=K$, that is $G \subseteq S$.

\section{Quotients of finite supergroups}

Let $G$ be an affine supergroup and let $H, N$ be supersubgroups of $G$ such that $H$ normalizes $N$. Denote the semi-direct product of $H$ and $N$ by $H \dot{\times} N$. More precisely, $(H \dot{\times} N)(A)=$ $H(A) \times N(A)$ and $(h, n)\left(h^{\prime}, n^{\prime}\right)=\left(h h^{\prime}, h^{\prime-1} n h^{\prime} n^{\prime}\right)$ for any $h, h^{\prime} \in H(A), n, n^{\prime} \in N(A), A \in$ $S A l g_{K}$. We have a natural morphism $g: H \dot{\times} N \rightarrow G,(h, n) \mapsto h n$. By Theorem 6.1 the image of $g$ is a closed supersubgroup of $G$ which is denoted by $H N$. The construction in Section 2 yields for any $A \in S A g_{K}$

$H N(A)=\left\{g \in G(A) \mid\right.$ there exists ff-covering $B$ of $A$ such that $\left.G\left(\iota_{A}^{B}\right)(g) \in H(B) N(B)\right\}$.

For example, assume that $N$ is a kernel of an epimorphism $\pi: G \rightarrow L$. The preimage $\left.\pi\right|_{H} ^{-1}\left(\left.\pi\right|_{H}(H)\right)$ coincides with $H N$. Theorem 6.1 and Corollary 6.1 imply that $I_{H N}=$ $K[G]\left(\pi^{*}\left(\operatorname{ker} \epsilon_{L}\right) \cap I_{H}\right)$.

Proposition 7.1 (see [4], part I, (6.2)) The quotient $H \tilde{\tilde{N}} / H$ is isomorphic to $N /(\tilde{\tilde{N}} \cap H)$.

Proof. The image of the canonical inclusion $(N /(N \cap H))_{(n)} \rightarrow(H N / H)_{(n)}$ is dense with respect to the Grothendieck topology of ff-coverings. In fact, if $g H(A) \in H N(A) / H(A)$, then there is a ff-covering $B$ of $A$ such that $g^{\prime} H(B)=h^{\prime \prime} n^{\prime \prime} H(B)=h^{\prime \prime} n^{\prime \prime}\left(h^{\prime \prime}\right)^{-1} H(B)$, where $g^{\prime}=G\left(\iota_{A}^{B}\right)(g), h^{\prime \prime} \in H(B), n^{\prime \prime} \in N(B)$. Therefore,

$$
N /(N \tilde{\tilde{N}} H)=(N /(N \tilde{\tilde{n}} H))_{(n)}=(H N \tilde{\tilde{\gamma}} H)_{(n)}=H \tilde{\tilde{N}} / H
$$

Lemma 7.1 Let $A$ be a finitely generated (commutative) superalgebra and I be a nilpotent superideal of $A$. If $\operatorname{dim} A / I$ is finite, then $A$ is finite dimensional.

Proof. Since $A_{1}$ is a finitely generated $A_{0}$-module, all we have to check is that $\operatorname{dim} A_{0}<\infty$. Denote by $V$ a finite dimensional subspace of $A_{0}$ such that $V+I_{0}=A_{0}$. Choose a nonnegative integer $k$ such that $I^{k+1}=0$. Let $I_{0}=\sum_{1 \leq i \leq l} A_{0} z_{i}$. For any $a \in A_{0}$ we have

$$
a=v+\sum_{1 \leq i \leq l} a_{i} z_{i} \text {, where } v \in V \text { and } a_{i} \in A_{0} .
$$


Repeating this procedure for the coefficients $a_{i}$ we obtain

$$
a=\sum_{0 \leq t \leq l k} \sum_{1 \leq i_{1}, \ldots, i_{t} \leq l} v_{i_{1}, \ldots, i_{t}} z_{i_{1}} \ldots z_{i_{t}}
$$

where $v, v_{i_{1}, \ldots, i_{t}} \in V$. Therefore $\operatorname{dim} A_{0} \leq \frac{l^{l k+1}-1}{l-1} \operatorname{dim} V$.

Corollary 7.1 An algebraic supergroup $G$ is finite iff $G_{r e d}$ is finite or iff $G_{\text {ev }}$ is finite.

For any finite supergroup $G$ denote by $|G|$ the dimension of $K[G]$. We call $|G|$ an order of $G$. By Lemma 6.2 of [27] there are pairwise-orthogonal idempotents $e_{1}, \ldots, e_{n} \in K[G]_{0}$ such that $\sum_{1 \leq i \leq n} e_{i}=1$ and each $K[G] e_{i}$ has a unique (nilpotent) maximal ideal $r e_{i}, r=$ $\operatorname{radK}[G]$. Without loss of generality one can assume that $\epsilon_{G}\left(e_{1}\right)=1$ and $\epsilon_{G}\left(e_{i}\right)=0$ for $i \geq 2$. A supersubalgebra of $K[G]$, generated by $e_{1}, \ldots, e_{n}$, is denoted by $B$.

Lemma 7.2 Any idempotent of $K[G]$ belongs to $B$.

Proof. Let $x=x_{0}+x_{1}$ be an idempotent in $K[G]$. The equality $x^{2}=x$ implies that $x_{0}$ is also an idempotent and $2 x_{0} x_{1}=x_{1}$. Multiplying the last equality by $x_{0}$ we obtain that $4 x_{0} x_{1}=x_{1}$, hence $x_{1}=0$. Any idempotent $x e_{i}$ belongs to $K[G] e_{i}$. In particular, $x e_{i}=\alpha e_{i}+y e_{i}$, where $\alpha=0,1$ and $y \in r$. On the other hand, $(x-\alpha) e_{i}$ is again an idempotent that equals to a nilpotent element $y e_{i}$. Hence any $x e_{i}$ is either zero or it is equal to $e_{i}$. Therefore $x=\sum_{1 \leq i \leq n} x e_{i} \in B$.

Lemma 7.3 The algebra $B$ is a Hopf (super)subalgebra.

Proof. We have to check that $B$ is a (super)subcoalgebra. The radical of the superalgebra $A=K[G] \otimes K[G]$ equals to $J=r \otimes K[N]+K[N] \otimes r$. Elements $e_{i} \otimes e_{j}$ are pairwise orthogonal idempotents and their sum equals $1 \otimes 1$. Furthermore, $A e_{i} \otimes e_{j} / J e_{i} \otimes e_{j} \simeq$ $K \otimes K \simeq K$. Applying Lemma 7.6 to the finite supergroup $G \times G$ we conclude that $\delta_{G}(B) \subseteq B \otimes B$.

Consider the natural epimorphism of supergroups $G \rightarrow S S p B$ and denote by $G^{(0)}$ its kernel. We can consider $G^{(0)}$ as a connected component of $G$. The equivalent definition will be given in Section 9 .

Assume that there is a finite supergroup $G$ and its supersubgroup $H$ such that $G \tilde{\tilde{F}} H$ is not affine. The pair $(G, H)$ is said bad. A bad pair defines a vector $v_{G, H}=(|G|,|G|-|H|) \in$ $\mathbf{N}^{2}$. Order vectors from $\mathbf{N}^{2}$ lexicographically from left to right. Choose a bad pair $(G, H)$ whose $v_{G, H}$ is minimal. Denote $K[G]^{H}$ by $R$.

By Theorem 5.2(ii) the property to be a faithfully exact supersubgroup is transitive. Therefore, there is not any supersubgroup $H^{\prime}$ such that $H<H^{\prime}<G$. On the other hand, the superideal $K[G] R^{+}+K[G] s_{G}\left(R^{+}\right)$is contained in $I_{H}$ and it is Hopf one. There is a supersubgroup $H^{\prime}$ such that $I_{H^{\prime}}=K[G] R^{+}+K[G] s_{G}\left(R^{+}\right)$. Since $H \leq H^{\prime}$ it follows that either $H=H^{\prime}$, that is $I_{H}=K[G] R^{+}+K[G] s_{G}\left(R^{+}\right)$, or $G=H^{\prime}$, that is $R=K$.

Assume that $E<G^{(0)}<G$. The minimality of $v_{G, H}$ and Proposition 7.1 imply that $H G^{(0)}<G$ and therefore, $H=H G^{(0)}$. Thus $G^{(0)} \leq H$. In the same way, by Proposition 6.4 we see that $G \tilde{\tilde{\gamma}} H$ is affine. Finally, if $G^{(0)}=E$, then $G$ is pure even and $G \tilde{\tilde{/}} H$ is always affine. The remaining case $G=G^{(0)}$ means that $K[G]$ is a local superalgebra with ker $\epsilon_{G}=r$. 
Lemma 7.4 The superalgebra $K[G]$ is a free $R$-module.

Proof. By Theorem $5.1 K[G]$ is a flat $R$-module. It remains to notice that $R^{+}$is a maximal nilpotent ideal of $R$ and use Corollary 2.1 [3], II, $\S 3$.

Lemma 7.5 Let $L$ be an affine supergroup and $N$ be an its supersubgroup such that $I_{N}=$ $K[L] T^{+}+K[L] s_{G}\left(T^{+}\right), T=K[L]^{N}$. Then, the canonical morphism $L \rightarrow S S p T$ induces an inclusion $L / N \subseteq S S p T$.

Proof. One has to check that the induced morphism $(L / N)_{(n)} \rightarrow S S p T$ is injective. Let $g_{1}, g_{2} \in L(A), A \in S A l g_{K}$, and assume that $\left.g_{1}\right|_{T}=\left.g_{2}\right|_{T}$. For a given $t \in T^{+}$we have $\delta_{L}(t)=t \otimes 1+\sum h_{1} \otimes t_{2}$, where $t_{2} \in T^{+}$. By definition,

$$
\begin{gathered}
\left(g_{1}^{-1} g_{2}\right)(t)=g_{1}\left(s_{L}(t)\right)+\sum g_{1}\left(s_{L}\left(h_{1}\right)\right) g_{2}\left(t_{2}\right)=g_{1}\left(s_{L}(t)\right)+\sum g_{1}\left(s_{L}\left(h_{1}\right)\right) g_{1}\left(t_{2}\right)= \\
g_{1}\left(s_{L}(t)+\sum s_{L}\left(h_{1}\right) t_{2}\right)=g_{1}\left(\epsilon_{L}(t)\right)=0 .
\end{gathered}
$$

Analogously,

$$
\begin{gathered}
\left(g_{1}^{-} 1 g_{2}\right)\left(s_{L}(t)\right)=g_{2}\left(s_{L}(t)\right)+\sum(-1)^{\left|h_{1}\right|\left|t_{2}\right|} g_{1}\left(t_{2}\right) g_{2}\left(s_{L}\left(h_{1}\right)\right)= \\
g_{2}\left(s_{L}(t)\right)+\sum(-1)^{\left|h_{1}\right|\left|t_{2}\right|} g_{2}\left(t_{2}\right) g_{2}\left(s_{L}\left(h_{1}\right)\right)=g_{2}^{-1}\left(\epsilon_{L}(t)\right)=0 .
\end{gathered}
$$

It follows that $g_{1}^{-1} g_{2}\left(I_{N}\right)=0$, that is $g_{1}^{-1} g_{2} \in N(A)$.

If $H=H^{\prime}$, then Lemma 7.5 implies that the morphism of $K$-functors $G \times H \rightarrow$ $G \times{ }_{S S p} R$ defined as $(g, h) \mapsto(g, g h), g \in G(A), h \in H(A), A \in S A l g_{K}$, is an isomorphism. Combining Proposition 4.2 with Lemma 7.4 we obtain that $G \tilde{\tilde{/}} H \simeq S S p R$ !

Let $R=K$. Since the ideal $I_{H}$ is nilpotent, one can repeat the arguments from Propositions 6.5 and 6.6 to conclude that $G=H$. Resuming all the above we obtain

Theorem 7.1 Let $G$ be a finite supergroup and $H \leq G$. Then, $G \tilde{\tilde{l}} H=S S p K[G]^{H}$.

Remark 7.1 In the conditions of the above theorem we have $G \tilde{\tilde{F}} H=G \tilde{/} H$. In fact, $K[G]^{H}$ is obviously noetherian and it remains to use Proposition 4.2.

\section{Brunden's question, $\operatorname{char} K=p>0$}

Let $G$ be an algebraic supergroup and $H$ be its supersubgroup such that $H_{e v}$ is reductive. It is equivalent to say that $H_{e v}$ is geometrically reductive [18, 19] and the last property is kept by taking quotients and normal subgroups [20]. As above, we assume that $K$ is algebraically closed. Let $A$ be a superalgebra. Denote by $A^{(n)}$ the superalgebra that coincides as a ring with $A$, but where each $a \in K$ acts as $a^{p^{-n}}$ does on $A$ (see [4]).

Lemma 8.1 (see [2], 3.1(a)) If $A$ is a Hopf superalgebra and char $K=p>0$, then the linear map $F^{n}: x \mapsto x^{p^{n}}$ (Frobenius morphism) is a Hopf superalgebra morphism $A^{(n)} \rightarrow A$. 
Proof. The identity $a^{p^{n}}=a_{0}^{p^{n}}$ for $a \in A$ implies that $F^{n}$ is a superalgebra morphism. Since $\delta_{A}$ and $s_{A}$ are superalgebra morphisms, the equations $s_{A} F^{n}=F^{n} s_{A}$ and $\delta_{A} F^{n}=$ $\left(F^{n} \otimes F^{n}\right) \delta_{A}$ follow easily.

Denote by $f_{n}: G \rightarrow S S p K[G]^{(n)}$ the morphism of supergroups dual to $F^{n}: K[G]^{(n)} \rightarrow$ $K[G]$. The kernel $G_{n}=\operatorname{ker} f_{n}$ is called the $n$-th infinitesimal supersubgroup. By Theorem $6.1, G_{n}$ is faithfully exact supersubgroup of $G$. Besides, $G / G_{n} \simeq S \operatorname{SpK}[G]_{0}^{p^{n}}$. If $G$ is algebraic, then any $G_{n}$ is finite.

Lemma 8.2 Let $L$ be an algebraic supergroup. For sufficiently large $t \geq 1$ the epimorphism $L \rightarrow L \tilde{/} L_{t}$ induces an epimorphism $L_{e v} \rightarrow L \tilde{/} L_{t}$. In particular, if $L_{e v}$ is reductive, then $L / L_{t}$ is also reductive.

Proof. One has to check tat $K[L]_{0}^{p^{t}} \cap K[L]_{1}^{2}=0$ for for some $t \geq 1$. Let $I$ be a radical of $K[L]_{0}$. The algebra $K[L]_{0}$ is noetherian. It follows that $I^{s}=0$ for some $s \geq 1$. If $W$ is a complement of vector subspace $I$ to $K[L]_{0}$, then $K[L]_{0}^{p^{t}}=W^{p^{t}}$, provided $p^{t} \geq s$. It remains to notice that $K[L]_{1}^{2} \subseteq I$ and $W^{p^{t}} \bigcap I=0$.

Proposition 8.1 The quotient $G \tilde{\tilde{/}} H$ is affine.

Proof. Consider the supersubgroup $H G_{t}$. By Lemma 3.1 one can assume that $H_{e v} \rightarrow$ $H \tilde{/} H_{t}=H \tilde{G_{t}} / G_{t}$ is an epimorphism. Combine with Corollary 4.5 from [21] we see that the quotient of $G / G_{t}$ over the supersubgroup $H G_{t} / G_{t}$ is affine. Since the property to be a faithfully exact supersubgroup is transitive we refer to Theorem 7.1 and Proposition 7.1 to conclude the proof.

\section{Quotients by normal supersubgroups, $\operatorname{char} K=0$}

An algebraic supergroup $G$ is called pseudoconnected if $\bigcap_{n \geq 0} \mathcal{M}^{n}=0$, where $\mathcal{M}=\operatorname{ker} \epsilon_{G}$.

Lemma 9.1 Let $G$ be an algebraic supergroup. The superideal $I=\bigcap_{n \geq 0} \mathcal{M}^{n}$ is a Hopf superideal and the supersubgroup $G^{[0]}=V(I)$ is normal and connected.

Proof. By definition, $s_{G}(\mathcal{M})=\mathcal{M}$. It remains to check that $I$ is a coideal and $\nu_{l}(I) \subseteq$ $I \otimes K[G]$. The trivial supersubgroup is obviously normal. In particular, $\nu_{l}(\mathcal{M}) \subseteq \mathcal{M} \otimes K[G]$ which implies $\nu_{l}\left(\mathcal{M}^{n}\right) \subseteq \mathcal{M}^{n} \otimes K[G]$ for all $n \geq 0$ and we conclude that $\nu_{l}(I) \subseteq I \otimes K[G]$. Furthermore,

$$
\delta_{G}\left(\mathcal{M}^{n}\right) \subseteq \sum_{0 \leq i \leq n} \mathcal{M}^{i} \otimes \mathcal{M}^{n-i} \subseteq \bigcap_{0 \leq i \leq n}\left(\mathcal{M}^{i} \otimes K[G]+K[G] \otimes \mathcal{M}^{n-i}\right)
$$

and

$$
\delta_{G}(I) \subseteq \bigcap_{n \geq 0} \delta_{G}\left(\mathcal{M}^{n}\right) \subseteq \bigcap_{n \geq 0}\left(\mathcal{M}^{n} \otimes K[G]+K[G] \otimes \mathcal{M}^{n}\right)=I \otimes K[G]+K[G] \otimes I .
$$

The supersubgroup $G^{[0]}$ is called a pseudoconnected component of $G$. It is clear that $G$ is pseudoconnected iff $G=G^{[0]}$. Additionally, $\operatorname{Dist}(G)=\operatorname{Dist}\left(G^{[0]}\right)$ and $\operatorname{Lie}(G)=$ $\operatorname{Lie}\left(G^{[0]}\right)$. The proof of Lemma 9.1 shows that if $N \unlhd G$, then $N^{[0]} \unlhd G$ also. Besides, an epimorphic image of a pseudoconnected supergroup is again pseudoconnected. 
Lemma 9.2 (Krull's intersection theorem) Let $A$ be a finitely generated commutative superalgebra and $V$ be a finitely generated $A$-supermodule. For any superideal $I$ of $A$ we have $\bigcap_{t \geq 0} I^{t} V=\left\{v \in V \mid\right.$ there exists $x \in I_{0}$ such that $\left.(1-x) v=0\right\}$.

Proof. Observe that $V$ is finitely generated as a $A_{0}$-module. Since $I^{t} \subseteq I_{0}^{\left[\frac{t}{2}\right]} \bigoplus I_{0}^{\left[\frac{t-1}{2}\right]} I_{1}$ we see that $I^{t} V \subseteq I_{0}^{\left[\frac{t-1}{2}\right]} V_{0} \oplus I_{0}^{\left[\frac{t-1}{2}\right]} V_{1}$. Proposition 5, [3], III, §3, concludes the proof.

Proposition 9.1 Let $\pi: G \rightarrow H$ is an epimorphism of algebraic supergroups. If char $K=$ 0 , then the induced short sequence of Lie superalgebras

$$
0 \rightarrow \operatorname{Lie}(\operatorname{ker} \pi) \rightarrow \operatorname{Lie}(G) \stackrel{d \pi}{\rightarrow} \operatorname{Lie}(H) \rightarrow 0
$$

is exact.

Proof. Since $I_{\operatorname{ker} \pi}=K[G] \pi^{*}\left(\operatorname{ker} \epsilon_{H}\right)$ it obviously implies that ker $d \pi=\operatorname{Lie}(\operatorname{ker} \pi)$. Combining Lemma 9.2 with word-by-word repeating the proof of Proposition 7.6, 4, part I, we obtain that $d \pi: \operatorname{Dist}(G) \rightarrow \operatorname{Dist}(H)$ is surjective. Now Lemma 3.1 concludes the proof.

Remark 9.1 If char $K=0$, then any algebraic supergroup $G$ is reduced (or smooth), meaning that the radical of $K[G]$ coincides with $I_{G_{e v}}$. Indeed, by Theorem 11.4 of [27] the Hopf superalgebra $K[G] / I_{G_{e v}}$ is reduced, and in particular, $G_{r}=G_{e v}$. It is proved in [28] that a completion of $K[G]$ with respect to a $\mathcal{M}$-adic topology is isomorphic to $K\left[\left[t_{1}, \ldots, t_{m} \mid z_{1}, \ldots z_{n}\right]\right]$ for any $\mathcal{M} \in \operatorname{Max}(K[G])$, where $m=\operatorname{dim} \operatorname{Lie}(G)_{0}, n=\operatorname{dim} \operatorname{Lie}(G)_{1}$ (see also Lemma 3.1 and use automorphism $T_{\mathcal{M}}$ from Theorem 5.1).

Any (left) $G$-supermodule $V$ is a $\operatorname{Dist}(G)$-supermodule via $\phi v=\sum(-1)^{|\phi|\left|v_{1}\right|} v_{1} \phi\left(f_{2}\right)$ for $\phi \in \operatorname{Dist}(G)$ and $v \in V, \tau_{V}(v)=\sum v_{1} \otimes f_{2}$, see [24, 25]. If $V, V^{\prime}$ are $G$-supermodules, then we have a canonical embedding $\operatorname{Hom}_{G}\left(V, V^{\prime}\right) \subseteq \operatorname{Hom}_{\operatorname{Dist}(G)}\left(V, V^{\prime}\right)$.

The proof of the following lemmas is a copy of the proofs of Proposition 7.5, Lemmas 7.15 and 7.16, 4], part I.

Lemma 9.3 Let $H_{1}, H_{2}$ be supersubgroups of an algebraic supergroup $G$ and $H_{1}$ be pseudoconnected. Then $H_{1} \subseteq H_{2}$ is equivalent to Dist $\left(H_{1}\right) \subseteq \operatorname{Dist}\left(H_{2}\right)$. Additionally, if charK $=0$, then $H_{1} \subseteq H_{2}$ is equivalent to Lie $\left(H_{1}\right) \subseteq \operatorname{Lie}\left(H_{2}\right)$.

Lemma 9.4 If $G$ is pseudoconnected and algebraic, then a supersubspace $W$ of a $G$ supermodule $V$ is a $G$-supesubmodule iff $W$ is a Dist $(G)$-supersubmodule. If char $K=0$, then $W$ is a $G$-supesubmodule iff $W$ is a Lie $(G)$-supersubmodule.

Lemma 9.5 If $G$ is pseudoconnected and algebraic, then $\operatorname{Hom}_{G}\left(V, V^{\prime}\right)=\operatorname{Hom}_{\operatorname{Dist}(G)}\left(V, V^{\prime}\right)$ for any $G$-supermodules $V$ and $V^{\prime}$. If char $K=0$, then $\operatorname{Hom}_{G}\left(V, V^{\prime}\right)=\operatorname{Hom}_{\text {Lie }(G)}\left(V, V^{\prime}\right)$.

Proposition 9.2 If $L$ is algebraic and $N \unlhd L$, then the equality Lie $(N)=\operatorname{Lie}(L)$ implies that $L \tilde{\tilde{/} N}=L \tilde{/} N$ is affine and finite. 
Proof. Without loss of generality, one can assume that $K[L]^{N}=K$ and $K$ is algebraically closed. Denote the supergroup $N \cap G_{\text {red }}$ by $U$. We have

$$
\operatorname{Lie}(U)=\operatorname{Lie}(N) \bigcap \operatorname{Lie}\left(G_{r e d}\right)=\operatorname{Lie}(G) \bigcap \operatorname{Lie}\left(G_{r e d}\right)=\operatorname{Lie}\left(G_{r e d}\right) .
$$

It follows that the affine group $\tilde{L_{r}} \tilde{U} / U$ is finite. In other words, an algebra $B=K\left[L_{\text {red }} / U\right]=$ $K\left[L_{r e d}\right]^{U}$ is finite dimensional. By Lemma 6.2 of [27] we have $B=\prod_{1 \leq i \leq n} B e_{i}^{\prime}$, where $e_{1}^{\prime}, \ldots e_{n}^{\prime}$ are pairwise orthogonal idempotents such that $\sum_{1 \leq i \leq n} e_{i}^{\prime}=1$. Besides, each algebra $B e_{i}^{\prime}$ is isomorphic to $K$. By Corollary 1, [3], II, $\S 4$, there are pairwise orthogonal idempotents $e_{1}, \ldots, e_{n} \in K[L]_{0}$ such that their respective images in $K\left[L_{r e d}\right]$ coincide with $e_{1}^{\prime}, \ldots e_{n}^{\prime}$ and $\sum_{1 \leq i \leq n} e_{i}=1$. Consider an idempotent $e=e_{i}$. As in Lemma 6.3 we have $\delta_{L}(e)-e \otimes 1 \in r \otimes K[L]+K[L] \otimes\left(r+I_{N}\right)$, where $r=\operatorname{rad} K[L]$. On the other hand, for any odd exponent $k$, the equality $\left(\delta_{L}(e)-e \otimes 1\right)^{k}=\delta_{L}(e)-e \otimes 1$ holds. For sufficiently large (odd) integer $k$ we infer $\delta_{L}(e)-e \otimes 1 \in K[L] \otimes I_{N}$, forcing $e \in K$. It obviously implies that $n=1, e_{1} \underset{\tilde{\tilde{N}}}{=} 1$ and $B=K$. Repeating the arguments from Propositions 6.5 and 6.6, we see that $L \tilde{/} N$ is affine. By Remark $6.1 R=K[L]^{N}$ is finitely generated and $L \tilde{\tilde{N}} N=L \tilde{/} N$. Let $I$ be a radical of $R$. Since $K[L]$ is a faithfully flat $R$-module we have $r \bigcap R \subseteq I=R \bigcap K[L] I \subseteq r$, that is $I=R \bigcap r$. In other words, the induced morphism $L_{\text {red }} \rightarrow(L \tilde{/} N)_{\text {red }}$ is an epimorphism. In particular, $(L \tilde{/} N)_{\text {red }} \simeq L_{\text {red }} / U$ is finite.

Corollary 9.1 For any algebraic supergroup $L$ the quotient $L / \tilde{L}^{[0]}$ is a finite supergroup.

Remark 9.2 If charK $=0$ and $\operatorname{Lie}\left(G_{e v}\right)=\operatorname{Lie}\left(N_{e v}\right)$, then all statements of Proposition 9.2 hold also.

We define a connected component of an algebraic group $G$ as the preimage of $\left(G / \tilde{G}^{[0]}\right)^{(0)}$ in $G$. It is not difficult to check that $G^{(0)}$ can be defined as the intersection of kernels of all morphisms $G \rightarrow L$, where $L$ is an even etale (super)group. Notice also that for any finite $G$ both components are the same. In fact, if $e_{1}, \ldots, e_{n}$ are all primitive idempotents of $K[G]$ and $\epsilon_{G}\left(e_{1}\right)=1$, then $\operatorname{ker} \epsilon_{G}=r+K[G] e$, where $e=\sum_{2 \leq i \leq n} e_{i}$ and $r=\operatorname{rad} K[G]$. Thus $I_{G^{[0]}}=K[G] e=I_{G^{(0)}}$.

Remark 9.3 The supersubgrop $G^{(0)}$ is an open subfunctor of $G$. In fact, if $e_{1}, \ldots, e_{n}$ are all primitive idempotents of $K[G]^{G^{[0]}}$, then $G^{(0)}=G_{e_{1}}$, provided $\epsilon_{G}\left(e_{1}\right)=1$.

Question 9.1 Does $G^{[0]}$ coincide with $G^{(0)}$ for arbitrary $G$ ?

Question 9.2 Is $G^{[0]}$ an open subfunctor for arbitrary $G$ ?

Lemma 9.6 If $G$ is pseudoconnected or connected, then Lie $(G)=0$ implies $G=E$. In particular, if char $K=0$ and $G$ is algebraic, then $G^{(0)}=G^{[0]}$.

Proof. In the above notations $\operatorname{Lie}(G)=0$ iff $r \subseteq K[G] e$, that is $\operatorname{ker} \epsilon_{G}=B e$ is an idempotent and nilpotent ideal simultaneously. As for the second statement, Proposition 9.1 implies $\operatorname{Lie}\left(G^{(0)} / G^{[0]}\right)=0$.

Lemma 9.7 If $H$ is a supersubgroup of $G$, then Lie $\left(N_{G}(H)\right)=(\operatorname{Lie}(G) / \operatorname{Lie}(H))^{\mathbf{A d}(H)}$. 
Proof. As in [6], II, $\S 5$, Lemma 5.7, it is enough to observe that $x \in \operatorname{Lie}\left(N_{G}(H)\right)(K)$ iff for any $A \in S A l g_{K}$ and $h \in H(A)$ we have

$$
\begin{aligned}
& e^{\varepsilon_{|x|} x^{\prime}} h^{\prime} e^{-\varepsilon_{|x|} x^{\prime}} h^{\prime-1}=e^{\varepsilon_{|x|}\left(x^{\prime}-\mathbf{A d}(h)\left(x^{\prime}\right)\right)} \in H\left(A\left[\varepsilon_{0}, \varepsilon_{1}\right]\right), \\
& e^{-\varepsilon_{|x|} x^{\prime}} h^{\prime} e^{\varepsilon_{|x|} x^{\prime}} h^{\prime-1}=e^{\varepsilon_{|x|}\left(\operatorname{Ad}(h)\left(x^{\prime}\right)-x^{\prime}\right)} \in H\left(A\left[\varepsilon_{0}, \varepsilon_{1}\right]\right),
\end{aligned}
$$

where $x^{\prime}=G\left(\iota_{K}^{A}\right)(x), h^{\prime}=G\left(p_{A}\right)(h)$. In other words, $x \in \operatorname{Lie}\left(N_{G}(H)\right)(K)$ iff $x^{\prime} \pm$ $\operatorname{Ad}(h)\left(x^{\prime}\right) \in \mathbf{L i e}(H)(A)$ for any superalgebra $A$.

Let us return to the situation of Section 6 , that is $N \leq G$, where $G$ is algebraic. As before, one can assume that $K$ is an algebraically closed of zero characteristic and, if it is necessary, that $K[G]^{N}=K$. Define the lower central (solvable) series of $L$ by $L^{1}=L, L^{i+1}=\left[L^{i}, L\right]$ (respectively, $L^{(0)}=L, L^{(i+1)}=\left[L^{(i)}, L_{\tilde{\tilde{F}}}^{(i)}\right]$.

As in Section 7 a pair $(G, N)$ is called bad, whenever $G \tilde{\tilde{F}} N$ is not affine. A vector $v \in \mathbf{N}^{2}$ is called positive iff at least one coordinate of $v$ is positive. Partially order the set of bad pairs by $(G, N)<\left(G^{\prime}, N^{\prime}\right)$ iff s $\operatorname{dim} \operatorname{Lie}\left(G^{\prime}\right)-\mathrm{s} \operatorname{dim} \operatorname{Lie}(G)$ is positive, otherwise $\mathrm{s} \operatorname{dim} \operatorname{Lie}\left(G^{\prime}\right)-\mathrm{s} \operatorname{dim} \operatorname{Lie}(G)=0$ and $\mathrm{s} \operatorname{dim} \operatorname{Lie}\left(N^{\prime}\right)-\mathrm{s} \operatorname{dim} \operatorname{Lie}(N)$ is positive. Choose a minimal bad pair $(G, N)$.

Proposition 9.3 If the superalgebra $L=\operatorname{Lie}(G)$ is not simple, then it is either semisimple, whose a unique proper ideal is $L^{2}$, or $L^{2}=0$.

Proof. Let $I$ be an proper ideal of $L$. Consider $L$ as $G$-supermodule via Ad : $G \rightarrow G L(L)$. By Lemma $9.4 I$ is a $G$-supersubmodule and we define the induced morphism $\operatorname{Ad}_{I}: G \rightarrow$ $G L(L / I)$. Denote ker $\mathbf{A d}_{I}$ by $H$. By Proposition $9.1 \mathrm{Lie}(H)$ is a proper supersubalgebra of $L$ iff $L^{2} \nsubseteq I$. As above, the minimality of $(G, N)$ and Propositions 6.4 and 7.1 imply that $L^{2}$ is a smallest (possibly zero) ideal of $L$. If $L$ is not semisimple, then considering the morphism $\left.A d\right|_{I}: G \rightarrow G L(I)$ for a proper abelian ideal $I$, we obtain $[L, I]=0$. In particular, $L^{3}=0$. Finally, repeating the above arguments for the morphism Ad we conclude that $L^{2}=0$.

Using Propositions 6.4 and 7.1 and Theorem 7.1 as well, one can always assume that $G$ is connected. If $L^{2}=0$, then $\operatorname{Dist}(G)$ is a commutative superalgebra. In its turn, $K[G]$ is cocommutative and $G$ is an abelian supergroup. In particular, $G_{e v} \unlhd G$ and the minimality arguments imply that $\operatorname{Lie}\left(G_{e v}\right)=0$. Corollary 7.1 and Theorem 7.1 conclude the proof in this case. If $L$ is simple, then $\operatorname{Lie}(N)=0$. By Proposition 6.4 and Lemma 9.6 this case is reduced to $N=N_{e v}$. The algebra $K[G]_{0}$ can be regarded as a coordinate algebra of an affine scheme on which $N$ acts on the right. Combining [6], III, $\S 2, \mathrm{n}^{\circ} 4$, with $K[G]^{N}=K$ we obtain that $K[G]_{0}$ is finitely dimensional. By Theorem 7.1 this case is also done.

Finally, if $L$ is semisimple, then we consider the supersubgroup $H=\left(G_{e v} N\right)^{[0]}$. Denote by $Q$ the normalizer $N_{G}(H)$ and by $D$ its Lie superalgebra. By the above, one can assume that $\operatorname{Lie}(N) \neq 0$. It follows that $L^{2} \subseteq \operatorname{Lie}(N)$. Denote $\operatorname{Lie}\left(G_{e v} N\right)=L_{0}+\operatorname{Lie}(N)$ by $M$. Since $L^{2} \subseteq M$ the supergroup $H$ acts trivially on $L / M$. By Lemma $9.7 D / M=$ $(L / M)^{\mathbf{A d}(H)}=L / M$ that infers $G=\underset{\sim}{Q}$ and $H \unlhd \underset{\tilde{\tilde{\sigma}}}{ }$. Again, by the minimality of $(G, N)$ we have either $G=G_{e v} N$ and then $G \tilde{\tilde{/}} N \simeq G_{e v} /\left(\tilde{G_{e v}} \cap N\right)$ is affine, or $L_{0} \subseteq \operatorname{Lie}(N)$ and Remark 9.2 concludes the proof of Theorem 6.2. 


\section{Two examples}

One more example of not necessary normal but faithfully exact supersubgroup is given by a Levi supersubgroup. In notations of Proposition 6.3, a Levi supersubgroup $L_{s, t}$ of $G=G L(m \mid n)$ consists of all matrices satisfying the equations $A_{12}=0, A_{21}=0, B_{12}=$ $0, B_{21}=0, B_{22}=0, C_{12}=0, C_{21}=0, C_{22}=0, D_{12}=0, D_{21}=0$. Additionally, the blocks $A_{22}$ and $D_{22}$ are diagonal matrices. It is clear that $L_{s, t} \simeq G L(s \mid t) \times T$, where $T$ is a torus of dimension $m+n-s-t$. Represent the coordinate superalgebra of $K[G L(m \mid n)]$ as $K[A, B, C, D]_{d_{1} d_{2}}$, where the blocks $A, B, C, D$ are identified with the sets of their coefficients, and $d_{1}=\operatorname{det}(A), d_{2}=\operatorname{det}(D)$.

Define the map $\pi: \underline{m+n} \rightarrow \underline{m+n}$ by the rule $\pi(i)=i+n$ and $\pi(j)=j-m$ for $1 \leq i \leq m<j \leq m+n$.

Lemma 10.1 There is a canonical isomorphism $\psi: G L(m \mid n) \rightarrow G L(n \mid m)$ such that $\psi\left(L_{s, t}\right)=L_{t, s}$.

Proof. Denote the matrix coordinate functions on $G L(m \mid n)$ by $a_{i j}$ and the similar functions on $G L(n \mid m)$ by $a_{i j}^{\prime}$. It is easy to see that $\psi^{*}\left(a_{i j}\right)=a_{\pi(i), \pi(j)}^{\prime}$ induces the required Hopf superalgebra isomorphism $K[G L(m \mid n)] \simeq K[G L(n \mid m)]$. In fact, only the equality $\psi^{*} s_{G L(m \mid n)}=s_{G L(n \mid m)} \psi^{*}$ is not trivial. It is enough to prove it for generators $a_{i j}$ and using the following formulaes

$$
\begin{gathered}
\psi^{*}\left(\begin{array}{cc}
A & B \\
C & D
\end{array}\right)=\left(\begin{array}{cc}
\psi^{*}(D) & \psi^{*}(C) \\
\psi^{*}(B) & \psi^{*}(A)
\end{array}\right), \\
s_{G L(m \mid n)}\left(\begin{array}{cc}
A & B \\
C & D
\end{array}\right)=\left(\begin{array}{cc}
\left(A-B D^{-1} C\right)^{-1} & -A^{-1} B\left(D-C A^{-1} B\right)^{-1} \\
-D^{-1} C\left(A-B D^{-1} C\right)^{-1} & \left(D-C A^{-1} B\right)^{-1}
\end{array}\right)
\end{gathered}
$$

it can be done by straightforward calculations.

Using Lemma 10.1 and transitivity of inducing functor it remains to prove that $L_{m, n-1}$ (or $L_{m-1, n}$ ) is faithfully exact. In what follows let $G=G L(m \mid n)$.

Lemma 10.2 The elements $a_{i, m+n} s_{G}\left(a_{m+n, j}\right)$ for $1 \leq i, j \leq m+n$, are $L=L_{m, n-1}$ invariants. Moreover, they generate a left coideal supersubalgebra $R$ such that $I_{L}=$ $K[G] R^{+}$.

Proof. Denote the image of $x \in K[G]$ in $K[L]$ by $\bar{x}$. Since

$\delta_{G}\left(a_{i, m+n} s_{G}\left(a_{m+n, j}\right)\right)=\sum_{1 \leq t, l \leq m+n}(-1)^{\left|a_{l j}\right|\left|a_{m+n, l}\right|+\left|a_{t, m+n} \|\right| a_{l j} \mid} a_{i t} s_{G}\left(a_{l j}\right) \otimes a_{t, m+n} s_{G}\left(a_{m+n, l}\right)$,

it follows that $R$ is a left coideal. Considering $K[G]$ as a $K[L]$-supercomodule (where $L$ acts on $G$ by the right multiplication) we determine

$$
\begin{aligned}
\tau_{K[G]}\left(a_{i, m+n} s_{G}\left(a_{m+n, j}\right)\right)= & a_{i, m+n} s_{G}\left(a_{m+n, j}\right) \otimes \overline{a_{m+n, m+n} s_{G}\left(a_{m+n, m+n}\right)}= \\
& a_{i, m+n} s_{G}\left(a_{m+n, j}\right) \otimes 1 .
\end{aligned}
$$

Notice that $a_{i, m+n} s_{G}\left(a_{m+n, j}\right)-\delta_{i, m+n} \delta_{m+n, j} \in I_{L}$ for $1 \leq i, j \leq m+n$ and consider an index $i \neq m+n$. We have $a_{i, m+n}=\sum_{1 \leq j \leq m+n} a_{i, m+n} s_{G}\left(a_{m+n, j}\right) a_{j, m+n} \in I_{L}$ and 
symmetrically, $s_{G}\left(a_{m+n, j}\right) \in I_{L}$ for $j \neq m+n$. Denote by $I$ a superideal generated by elements $a_{i, m+n}, s_{G}\left(a_{m+n, j}\right)$ for $1 \leq i, j<m+n$. It is obvious that $L \subseteq V(I)$. On the other hand, $g \in V(I)(A)$ iff $g \in \operatorname{Stab}_{G}\left(K v_{m+n}\right)(A)$ and $g^{-1} \in \operatorname{Stab}_{G}\left(\sum_{1 \leq i \leq m+n-1} K v_{i}\right)(A)$. The superversion of [4], part I (1.4) completes the proof of this claim.

By Proposition 5.2 and Theorem 5.1 all we have to show is that $K[G] \mathcal{M} \neq K[G]$ for all $\mathcal{M} \in \operatorname{Max}(R)$. Using the reduction from Corollary 5.1 one can work with algebraic groups $L_{0, n-1} \subseteq G L(0 \mid n) \simeq G L(n)$. In other words, we can set $G=G L(n), L=$ $\operatorname{Stab}_{G}\left(K v_{n}\right) \cap \operatorname{Stab}_{G}\left(\sum_{1 \leq i \leq n-1} K v_{i}\right)$. By Corollary 4.5 of [21], the quotient $G / L$ is affine and it is isomorphic to $S p_{K} K[G]^{L}$. In particular, $K[G]^{L} \leq K[G]$. We will show that $K[G]^{L}$ is generated by the elements $a_{i n} s_{G}\left(a_{n j}\right)$ for $1 \leq i, j \leq n$.

Consider an element $\frac{f}{d^{k}} \in K[G]$, where $f \in K\left[a_{i j} \mid 1 \leq i, j \leq n\right]$ and $d=\operatorname{det}\left(a_{i j}\right)$. Represent $f$ as

$$
f=\sum_{\alpha \in \mathbf{N}^{n}} f_{\alpha} \prod_{1 \leq i \leq n} a_{i n}^{\alpha_{i}}, \text { where } f_{\alpha} \in K\left[a_{i j} \mid 1 \leq i \leq n, 1 \leq j \leq n-1\right] .
$$

It can be checked easily that $\frac{f}{d^{k}} \in K[G]^{L}$ iff all monomials $\prod_{1 \leq i \leq n} a_{i n}^{\alpha_{i}}$ in the above representation of $f$ have degree $k$ and all coefficients $f_{\alpha}$ are $G L(n-1)$-semi-invariants of weight $k$ with respect to the action of this group by right multiplications on the variety of $n \times(n-1)$-matrices $M_{n \times(n-1)}$. By Igusa's Theorem (see [30] and Corollary 3.5 of [31]) the algebra $K\left[M_{n \times(n-1)}\right]^{S L(n-1)}=K\left[a_{i j} \mid 1 \leq i \leq n, 1 \leq j \leq n-1\right]^{S L(n-1)}$ is generated by all minors of size $n-1$ which are just semi-invariants $s_{G}\left(a_{n j}\right) d$ of weight 1 .

The next example shows that a quotient $X \tilde{\tilde{/}} G$ (as well $X \tilde{X} G$ ) is not always affine even if $G$ is finite. In what follows $\operatorname{char} K=0$. Let $G=G_{a}^{-}$be an odd unipotent supergroup such that $K[G]=K[t]$, where $|t|=1, \delta_{G}(t)=t \otimes 1+1 \otimes t$ and $\epsilon_{G}(t)=0, s_{G}(t)=-t$. A superspace $V$ is a $G$-supermodule iff there is $\phi \in \operatorname{End}_{K}(V)_{1}, \phi^{2}=0$, such that $\tau_{V}(v)=$ $v \otimes 1+\phi(v) \otimes t$. Moreover, $V^{G}=\operatorname{ker} \phi$. Assume that $\mathrm{s} \operatorname{dim} V=(1,1)$ and $v_{1}, v_{2}$ form a $\mathbf{Z}_{2}$-homogeneous basis of $V$. Set $\phi\left(v_{1}\right)=v_{2}, \phi\left(v_{2}\right)=0$. The symmetric superalgebra $S(V)$ has the induced $G$-supermodule structure by

$$
\tau_{S(V)}\left(v_{1}^{r}\right)=v_{1}^{r} \otimes 1+r v_{1}^{r-1} v_{2} \otimes t, \tau_{S(V)}\left(v_{1}^{r-1} v_{2}\right)=v_{1}^{r-1} v_{2} \otimes 1, r \geq 0 .
$$

Since $\tau_{S(V)}$ is a superalgebra morphism, there is an affine scheme $X$ such that $K[X]=S(V)$ and $G$ acts on $X$. Denote $K[X]^{G}$ by $R$. We have $R_{0}=K, R_{1}=\sum_{t \geq 0} K v_{1}^{t} v_{2}$ and $R_{1}^{2}=0$. In particular, $R$ is commutative as an algebra.

Lemma 10.3 Any flat $R$-supermodule is flat as a module.

Proof. Any exact sequence of $R$-modules $0 \rightarrow V \rightarrow W \rightarrow U \rightarrow 0$ can be turn into an exact sequence of $R$-supermodules. In fact, set $V_{1}=V \cap R_{1} W$ and let $V_{0}$ be a complement of $V_{1}$ to $V$. Since $V_{0} \bigcap R_{1} W=0$, then $R_{1} W$ has a complement $W_{0}$ to $W$ such that $V_{0} \subseteq W_{0}$. Finally, $U=W_{0} / V_{0} \bigoplus W_{1} / V_{1}$. If $M$ is a flat $R$-supermodule, then the functor $M \otimes_{R}$ ? takes our sequence (of supermodules!) to an exact one.

Assume that $X \tilde{/} G$ or $X \tilde{\tilde{\mu}} G$ is an affine superscheme. By Proposition $4.1 S(V)$ is a faithfully flat $R$-supermodule. Combining Lemma 10.3 with Corollary 2.1 [3], II, $\S 3$, we obtain that $S(V)$ is a free $R$-module. Let $R f \simeq R, f \in S(V)$. The equality $R f=R f_{0}+K f_{1}$ implies that either $R f=R f_{0}$ or $K f_{1}$ is a projective $R$-module. Again by Corollary 2.1 
[3], II, $\S 3, K f_{1}$ has to be free, but it is obviously impossible. Thus $S(V)=\bigoplus R f$, where $f$ runs over a basis of $S(V)_{0}$. Without loss of generality one can assume that some $f$ equals 1 . On the other hand, the summand $R$ has nontrivial intersections with all other summands! This contradiction shows that both $X \tilde{/} G$ and $X \tilde{\tilde{\mu}} G$ are not affine.

\section{Acknowledgements}

The author thanks FAPESP (proc. 07/54834-9) for the support during his visit to Sao Paulo University. This work was also partially supported by RFFI 07-01-00392. Special thanks to Professor Alexandr Grishkov for his invitation and to Professor Jonathan Brundan for bringing to my attention the example of Levi supersubgroup and communicating to me interesting questions.

\section{References}

[1] A.N.Zubkov, Some properties of general linear supergroups and of Schur superalgebras, (Russian), Algebra Logika, 45(2006), N 3, 257-299.

[2] N.J.Muir, Polynomial representations of the general linear Lie superalgebras, Ph.D. Thesis, University of London, 1991.

[3] N.Bourbaki, Commutative Algebra, Addison-Wesley, 1972.

[4] J.Jantzen, Representations of algebraic groups, Academic Press, Inc., 1987.

[5] I.Bucur and A.Deleanu, Introduction to the theory of categories and functors, A WileyInterscience Publ.,1968.

[6] M.Demazure and P.Gabriel, Groupes algebriques I, Paris/Amsterdam, 1970.

[7] S.I.Gelfand and Yu.I.Manin, Homological algebra, Algebra, V, Encyclopaedia Math. Sci. 38, 1994.

[8] D.Leites, Introduction to the theory of supermanifolds, (Russian), Uspekhi Mat.Nauk, 35(1980), N1(211), 3-57.

[9] B.Parshall and J.Wang, Quantum linear groups, Mem.Amer.Math. Soc., 439, 1991.

[10] A.Masuoka and D.Wigner, Faithfull flatness of Hopf algebras, J.Algebra, 170(1994), 156-164.

[11] M.Takeuchi, A correspondence between Hopf ideals and sub-Hopf algebras, Manuscripta Math., 7(1972), 251-270.

[12] M.Takeuchi, Relative Hopf modules - Equivaleces and freeness criteria, J.Algebra, 60(1979), 452-471.

[13] M.Takeuchi, A note on geometrically reductive groups, J.Fac.Sci.Univ.Tokyo, Sec. IA, 20(1973), 387-396.

[14] M.Takeuchi, Formal schemes over fields, Comm. Algebra, 5(14), 1977, 1483-1528. 
[15] F. Kasch, Modules and rings, LMS Monographs, 17, Academic Press, Inc., 1982.

[16] F.A.Berezin, Introduction to algebra and analysis with anticommuting variables, (Russia), Moskov.Gos.Univ., Moscow, 1983.

[17] J.E.Humphreys, Linear algebraic groups, Springer-Verlag, 1975.

[18] W.J. Haboush, "Reductive groups are geometrically reductive" Ann. of Math. , 102(1975), 67-83.

[19] C.S. Seshadri, "Geometric reductivity over arbitrary base" Adv. Math. , 26(1977), $225-274$.

[20] H.Borsari and W.F.Santos, Geometrically reductive Hopf algebras, J.Algebra, 152(1992), 65-77.

[21] E.Cline, B.Parshall and L.Scott, Induced modules and affine quotients, Math.Ann., 230(1977), 1-14.

[22] J.Igusa, On the arithmetic normality of the Grassman variety, Proc.Natl.Acad.Sc. USA, 40(1954), 309-313.

[23] C. de Concini, D.Eisenbud and C.Procesi, Young diagrams and determinantal varieties, Invent.Math., 56(1980), 129-165.

[24] J.Brundan and J.Kujawa, A new proof of the Mullineux conjecture, J. Algebraic Combin., 18(2003), 13-39.

[25] J.Brundan and A.Kleshev, Modular representations of the supergroup $Q(n), I$, J.Algebra.

[26] M.Scheunert, The theory of Lie superalgebras, Lect. Notes in Math., 716(1979).

[27] W.C.Waterhouse, Introduction to affine group schemes, Springer-Verlag, 1979.

[28] R.Fioresi, Smoothness of algebraic supervarieties and supergroups, arXiv : math/0703491v1.

[29] L.Caston and R.Fioresi, Mathematical foundations of supersymmetry, arXiv : math/0710.5742v1.

[30] J.Igusa, On the arithmetic normality of the Grassman variety, Proc.Natl.Acad.Sc. USA, 40(1954), 309-313.

[31] C. de Concini, D.Eisenbud and C.Procesi, Young diagrams and determinantal varieties, Invent.Math., 56(1980), 129-165. 